Review / meta-analyses

\title{
Burnout in mental health professionals: A systematic review and meta-analysis of prevalence and determinants
}

\author{
Karen O'Connor ${ }^{\mathrm{a}, *}$, Deirdre Muller Neff ${ }^{\mathrm{a}}$, Steve Pitman ${ }^{\mathrm{b}}$ \\ a Department of Psychiatry, University College Cork, Ireland \\ ${ }^{\mathrm{b}}$ Institute of Leadership, Royal College of Surgeons of Ireland, Ireland
}

\section{A R T I C L E I N F O}

\section{Article history:}

Received 20 April 2018

Received in revised form 12 June 2018

Accepted 13 June 2018

Available online 26 June 2018

\section{Keywords:}

Burnout

Stress

Compassion fatigue

Morale

Mental health professionals

\begin{abstract}
A B S T R A C T
This study aimed to estimate the level of burnout in mental health professionals and to identify specific determinants of burnout in this population. A systematic search of MEDLINE/PubMed, PsychINFO/Ovid, Embase, CINAHL/EBSCO and Web of Science was conducted for original research published between 1997 and 2017. Sixty-two studies were identified as meeting the study criteria for the systematic review. Data on the means, standard deviations, and prevalence of the dimensions of burnout were extracted from 33 studies and included in the meta-analysis $(n=9409)$. The overall estimated pooled prevalence for emotional exhaustion was 40\% (CI 31\%-48\%) for depersonalisation was $22 \%$ (CI $15 \%-29 \%$ ) and for low levels of personal accomplishment was 19\% (CI 13\%-25\%). The random effects estimate of the mean scores on the Maslach Burnout Inventory indicate that the average mental health professional has high levels of emotional exhaustion [mean 21.11 (95\% CI 19.98, 22.24)], moderate levels of depersonalisation [mean $6.76(95 \%$ CI 6.11, 7.42)] but retains reasonable levels of personal accomplishment [mean 34.60 (95\% CI 32.99, 36.21)]. Increasing age was found to be associated with an increased risk of depersonalisation but also a heightened sense of personal accomplishment. Work-related factors such as workload and relationships at work, are key determinants for burnout, while role clarity, a sense of professional autonomy, a sense of being fairly treated, and access to regular clinical supervision appear to be protective. Staff working in community mental health teams may be more vulnerable to burnout than those working in some specialist community teams, e.g., assertive outreach, crisis teams.
\end{abstract}

(c) 2018 Elsevier Masson SAS. All rights reserved.

\section{Introduction}

The novelist Graham Greene first introduced the term 'burnt out' when he wrote about a fictional architect who could no longer find meaning in art or pleasure in life [1]. The term 'burnout' was introduced to the scientific literature in 1974 by an American psychologist Herbert J Freudenberger where he described burnout as a 'state of mental and physical exhaustion caused by one's professional life' [2]. Freudenberger defined it as something that related exclusively to frontline human service workers. Subsequently, Maslach and Jackson defined burnout as a psychological syndrome that occurs in professionals who work with other people in challenging situations that is characterised by (a) emotional exhaustion; feeling overburdened and depleted of emotional and physical resources, (b) depersonalisation; a negative and cynical

\footnotetext{
* Corresponding author at: Department of Psychiatry, Cork University Hospital, Cork, Ireland.

E-mail address: Karen.oconnor3@hse.ie (K. O’Connor).
}

attitude towards people, and (c) a diminished sense of personal accomplishment $[3,4]$. Although, this definition of burnout remains most prominent in the literature other definitions of burnout have also been proposed [5]. Kirstensen et al. 2005 proposed that fatigue and exhaustion are the core feature of burnout but that depersonalisation is a coping strategy, while reduced personal accomplishment a consequence rather than a defining feature of burnout [5]. Demerouti and Bakker (2007), proposed that burnout was defined by two core dimensions (a) affective, physical and cognitive exhaustion and (b) disengagement from work [6]. An important development in this field has been an attempt by researchers to expand their understanding of burnout by looking at what could be considered its positive antithesis which has been defined as 'work engagement' [7,8]. However, while some researchers consider engagement to be the opposite of burnout [7]. Others define engagement as a persistent, positive affective-motivational state of contentment that is characterised by the three components of vigour, dedication and absorption. In this view, work engagement is an independent and distinct concept, which is not the opposite of burnout [9]. 
Burnout has been found to be associated with job dissatisfaction, low organisational commitment, absenteeism, intention to leave the job, and turnover [7,10]. Furthermore, there is considerable evidence that burnout has negative impacts on the physical and mental well-being of the individual worker [11], the welfare and functioning of the team and organisation in which they work [12,13], and is associated with lower productivity and impaired quality of care provided to patients [14]. Factors particular to the mental health field have been proposed to make workers in this field more vulnerable to burnout [7]. These factors include stigma of the profession [15], demanding therapeutic relationships [15] and threats of violence from patients and patient suicide $[15,16]$. However, a systematic review and meta-analysis of the prevalence and determinants of burnout in MHPs has not been conducted.

\subsection{Aims of this study}

The aim of this review is [1] to quantify the level of burnout in MHPs and [2] to identify specific determinants of burnout in MHPs.

\section{Methods}

\subsection{Literature search}

We used the PRISMA guidelines. A systematic search of MEDLINE/PubMed, PsychINFO/Ovid, Embase, CINAHL/EBSCO and Web of Science was conducted in May 2017 for original research published from $1^{\text {st }}$ January 1997 until $31^{\text {st }}$ December 2016. Relevant controlled vocabulary terms and free text terms related to burnout and MHPs were used to search each database. In all databases, the search was restricted to studies published in English. All studies had to be published in a peer-reviewed journal. The reference lists from articles and reviews were examined for any additional studies. The full search strategies for the individual databases can be found in Appendix 1 .

\subsubsection{Inclusion and exclusion criteria}

The inclusion criteria were [1]: the study examined the prevalence/ determinants of burnout [2], the sample population was comprised of MHPs (including doctors, nurses, social workers, psychologists, occupational therapists, counsellors) working in mental health services [3], the study had to be empirical and quantitative [4] the response rate was greater than $25 \%$ [5], the study sample was comprised of at least 50\% MHPs [6], the study included at least 50 participants. The exclusion criteria was [1] the study did not use a validated measure of burnout.

\subsubsection{Study selection, data extraction and assessment of study quality}

After removing the duplicates, two investigators (KOC and DMN) reviewed study titles and abstracts for eligibility. If at least one of them considered an article as potentially eligible, the full texts were assessed by the same reviewers. Any disagreements were resolved by discussion. Detailed information on the country, data source, study population, and results were extracted from each included study into a standardized spreadsheet by one author and checked by a second author (KOC and DMN). EndNote X7.3.1 (Thomas Reuters, New York, USA) was used to organize the identified articles.

Two investigators (KOC and DMN) independently assessed the risk of bias of each of the included studies. A score for quality, modified from the Newcastle-Ottawa Scale (NOS), was used to assess the appropriateness of research design, recruitment strategy, response rate, representativeness of the sample, objectivity/reliability of outcome determination, power calculation provided, and appropriate statistical analyses (See Appendix 2). Score disagreements were resolved by consensus. An NOS score of
8 or more was considered 'good,' a score of 5 or less was considered 'poor.'

\subsection{Data synthesis}

The meta-analyses were conducted using Comprehensive Meta-Analysis software, version 3 (Biostat Inc., NJ, USA). In light of expected differences in study sample and design, randomeffects models were used to calculate the pooled means and prevalence. Heterogeneity across studies was tested using Q statistics [17], and the $\mathrm{I}^{2}$ [18]. Results from studies grouped according to pre-specified study-level characteristics were compared using subgroup analyses (for MBI-HSS High EE/DP/PA 'cut off score, geographical location and NOS) and random effects meta-regression (for age, sex, study size and professional background of participants). To address the issue of publication bias, we examined funnel plots [19], and used the Eggers Test [20].

\section{Results}

\subsection{Search outcome}

The electronic literature search identified 1348 unique citations. Based on a review of article titles and abstracts 1262 citations were excluded. After full-text review 62 articles remained (See Fig. 1 for PRISMA flow diagram). The features of the identified studies are summarised in Table 1.

\subsection{Study population and study design}

Studies conducted across 33 different countries were identified. The vast majority of studies were cross-sectional $(N=57)$ and multi-site $(\mathrm{N}=47)$. However, five studies had a longitudinal design with follow-up times varying between six months $[67,68]$ and five years [50]. Self-reported questionnaires were utilised in every study. The number of respondents ranged from 60 [36] to 2258 [45]. The mean study size was 370.61 (SD 457.77), the median was 195. In most studies, female respondents were over-represented. Mean age of respondents ranged from 30.9 years [39] to 51.6 years old [71] and the response rate varied between 26\% [16] and 100\% [28]. The minority of studies $(\mathrm{N}=11)$ examined burnout in the inpatient setting exclusively. The rest examined burnout in community settings or a mix of community and inpatient settings.

Most studies examined the prevalence and correlates of burnout in several different MHP groups $(\mathrm{N}=31)$. Data on burnout in nursing staff was gathered in 30 studies, in doctors in 17 studies, in psychologists in ten studies, in occupational therapists in eight studies, in social workers in 12 studies. Although the data on individual professional groups was not reported in each of these studies.

\subsection{Quality of studies}

On the modified Newcastle-Ottawa Scale (NOS) 15 of the studies rated as being of good quality (score $\geq 8$ ) 41 studies rated as being of moderate quality (score 6-7) and six studies rated as being of poor quality (score $\leq 5)$ [36] (See Table 1)

\subsection{Measurement of burnout}

Eight validated measures of burnout are cited in the literature between 1997 and 2017. These are the Maslach Burnout Inventory (MBI) [83] $(\mathrm{n}=54)$, the Oldenburg Burnout Inventory (OLBI) [6] $(n=2)$, the Copenhagen Burnout Inventory (CBI) [5]( $n=3)$, Pines Burnout Measure $(n=3)$, the Psychologists Burnout Inventory $(\mathrm{n}=2)$, the Organisational Social Context Scale (OSCS) [84] $(\mathrm{n}=1)$, 


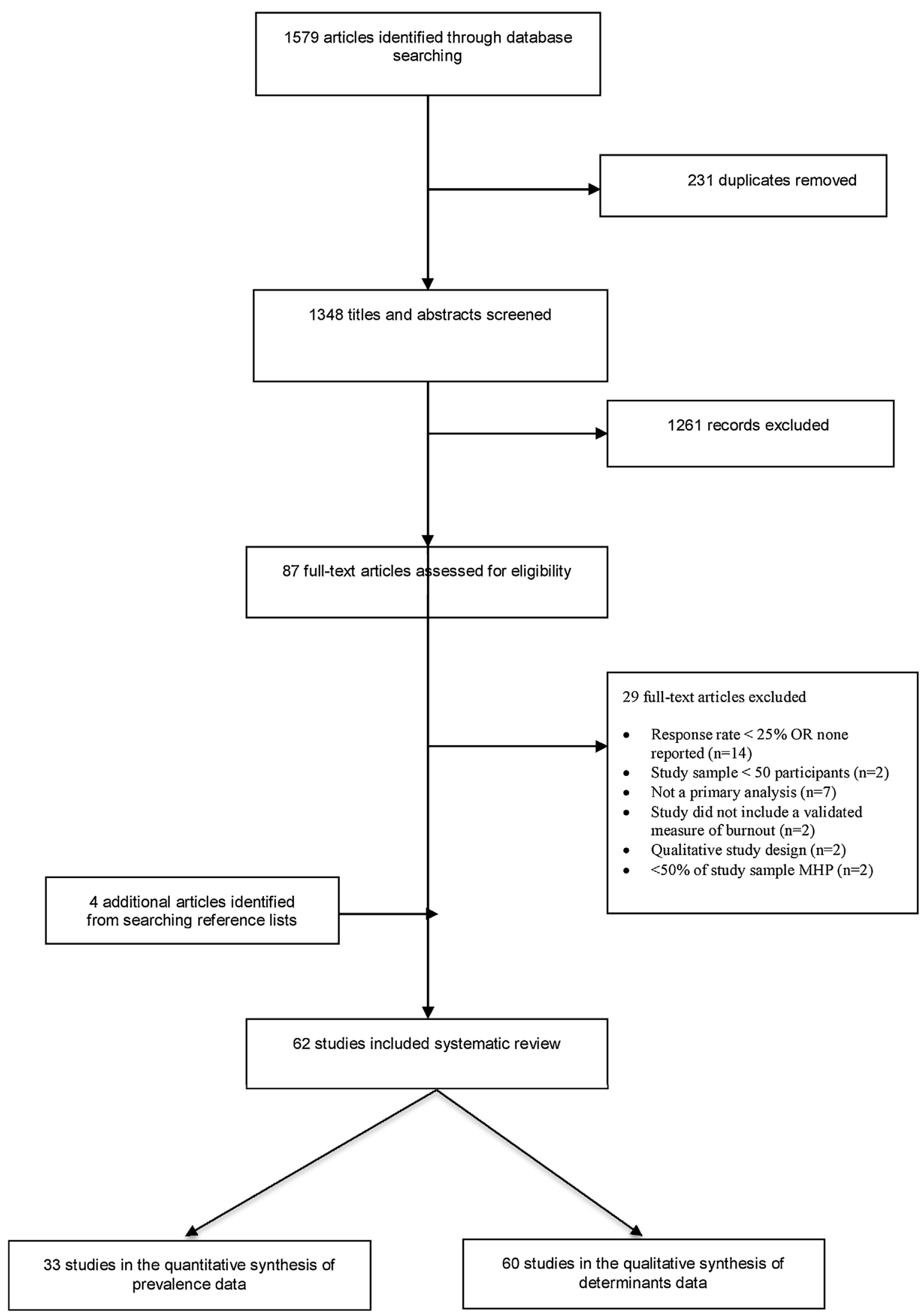

Fig. 1. Preferred Reporting Items for Systematic Reviews and Meta-Analyses (PRISMA) flow diagram. 
Table 1

Overview of the selected studies, the basic characteristics and results.

\begin{tabular}{|c|c|c|c|c|c|c|c|c|c|c|}
\hline Study & Design & $\begin{array}{l}\text { Study } \\
\text { population }\end{array}$ & $\begin{array}{l}\text { Response } \\
\text { rate }\end{array}$ & $\begin{array}{l}\text { Sample size } \\
\text { and } \\
\text { characteristics }\end{array}$ & $\begin{array}{l}\text { Burnout } \\
\text { measure }\end{array}$ & $\mathrm{EE}$ & DP & PA & $\begin{array}{l}\text { Burnout } \\
\text { Prevalence } \\
\text { High EE }>21 \\
\text { High DP }>8 \\
\text { Low PA }<28\end{array}$ & $\begin{array}{l}\text { Quality } \\
\text { Score } \\
\text { Good } \geq 8 \\
\text { Moderate } \\
6-7 \\
\text { Poor } \leq 5\end{array}$ \\
\hline $\begin{array}{l}\text { [21] } \\
\text { Angermeyer et al. } \\
2006 \\
\text { Germany }\end{array}$ & $\mathrm{CS}$ & $\begin{array}{l}\text { Nursing staff } \\
\text { from } 5 \\
\text { psychiatric } \\
\text { facilities in } \\
\text { Leipzig and } \\
\text { nearby. }\end{array}$ & $48.3 \%$ & $\begin{array}{l}\mathrm{N}=140 \\
\text { Male: } 18.7 \% \\
\text { Female: } 81.3 \% \\
\text { Mean age: } 38.9 \\
\text { years }\end{array}$ & MBI- HSS & $14.73(8.75)$ & $\begin{array}{l}5.73 \\
(5.08)\end{array}$ & $34.87(7.53)$ & NR & 7 \\
\hline $\begin{array}{l}\text { [22] } \\
\text { Ashtari et al. } 2009 \\
\text { Iran }\end{array}$ & $\mathrm{CS}$ & $\begin{array}{l}\text { Mental Health } \\
\text { Professionals } \\
\text { from large } \\
\text { psychiatric } \\
\text { hospital in } \\
\text { Tehran } \\
\text { (1370 inpatient } \\
\text { beds) }\end{array}$ & $95 \%$ & $\begin{array}{l}\mathrm{N}=100 \\
\text { Nurses }(\mathrm{N}=22) \\
\text { co-nurses } \\
(\mathrm{N}=29) \\
\text { Psychologists } \\
(\mathrm{N}=31) \\
\text { Social workers } \\
(\mathrm{N}=12) \\
\text { Occupational } \\
\text { therapists } \\
(\mathrm{N}=6) \\
\text { Male: } 32 \% \\
\text { Female: } 67 \% \\
\text { Mean age: } 38.9 \\
\text { years }\end{array}$ & MBI- HSS & $\begin{array}{l}29.4 \\
(6.9)\end{array}$ & $\begin{array}{l}9.3 \\
(2.1)\end{array}$ & $34.5(7.0)$ & $\begin{array}{l}\text { High EE: } 42.5 \% \\
\text { High DP: } 65.5 \% \\
\text { Low PA: } 21 \% \\
\text { Cut off points } \\
\text { for 'high' } \\
\text { unclear. }\end{array}$ & 7 \\
\hline $\begin{array}{l}\text { [23] } \\
\text { Benbow and Jolley, } \\
2002 \\
\text { UK }\end{array}$ & $\mathrm{CS}$ & $\begin{array}{l}\text { Consultant old } \\
\text { age psychiatrist } \\
\text { from across UK }\end{array}$ & $31.66 \%$ & $\begin{array}{l}\mathrm{N}=145 \\
\text { Male: } 66.9 \% \\
\text { Female: } 33.1 \%\end{array}$ & MBI-HSS & $\begin{array}{l}31.26 \\
(10.99)\end{array}$ & $\begin{array}{l}7.06 \\
(5.62)\end{array}$ & $\begin{array}{l}29.74 \\
(6.15)\end{array}$ & NR & 6 \\
\hline $\begin{array}{l}\text { [24] } \\
\text { Bilings et al, } 2003 \\
\text { UK }\end{array}$ & $\mathrm{CS}$ & $\begin{array}{l}\text { Assertive } \\
\text { Outreach } \\
\text { (AOT) } \\
\text { and } \\
\text { Community } \\
\text { Mental Health } \\
\text { Teams (CMHT) }\end{array}$ & $82.9 \%$ & $\begin{array}{l}\mathrm{N}=301 \\
\text { Male: } 46.18 \% \\
\text { Female: } 53.82 \%\end{array}$ & MBI-HSS & $\begin{array}{l}\text { AOT } \\
17.4(\mathrm{NR}) \\
\text { CMHT } \\
19.0 \\
(\mathrm{NR})\end{array}$ & $\begin{array}{l}\text { AOT } \\
4.4(\mathrm{NR}) \\
\text { CMHT } \\
5.7 \\
(\mathrm{NR})\end{array}$ & $\begin{array}{l}\text { AOT } \\
34.8(\mathrm{NR}) \\
\text { CMHT } \\
32.7(\mathrm{NR})\end{array}$ & NR & 8 \\
\hline $\begin{array}{l}\text { [25] } \\
\text { Blau et al. } 2013 \\
\text { USA }\end{array}$ & $\mathrm{CS}$ & $\begin{array}{l}\text { Psychiatric } \\
\text { rehabilitation } \\
\text { practitioners }\end{array}$ & $44.6 \%$ & $\begin{array}{l}\mathrm{N}=1639 \\
\text { Male: } 27 \% \\
\text { Female: } 73 \% \\
\text { Psychologists } \\
(\mathrm{N}=361) \\
\text { Social workers } \\
(\mathrm{N}=246) \\
\text { Average age of } \\
41-50 \text { years old } \\
\text { Average length } \\
\text { of service } 10 \\
\text { years }\end{array}$ & MBI-HSS & NR & NR & NR & NR & 8 \\
\hline $\begin{array}{l}\text { [26] } \\
\text { Bowers et al. } 2009 \\
\text { UK }\end{array}$ & $\mathrm{CS}$ & $\begin{array}{l}\text { Nursing staff on } \\
136 \\
\text { acute } \\
\text { admission } \\
\text { psychiatric } \\
\text { wards in } \\
\text { England }\end{array}$ & $56 \%$ & $\begin{array}{l}\mathrm{N}=1525 \\
\text { Nurse } 67 \% \\
\text { Healthcare } \\
\text { assistants } 29 \% \\
\text { Male: } 33 \% \\
\text { Female: } 66 \%\end{array}$ & MBI- HSS & $\begin{array}{l}17.78 \\
(11.39)\end{array}$ & $\begin{array}{l}5.49 \\
(5.09)\end{array}$ & $35.46(8.16)$ & NR & 8 \\
\hline $\begin{array}{l}\text { [27] } \\
\text { Bressi et al. } 2009 \\
\text { Italy }\end{array}$ & $\mathrm{CS}$ & $\begin{array}{l}\text { Psychiatrists } \\
\text { working in } \\
\text { Italian public } \\
\text { health system } \\
\text { in Milan }\end{array}$ & $70 \%$ & $\begin{array}{l}\mathrm{N}=81 \\
\text { Male: } 42 \% \\
\text { Female: } 58 \% \\
\text { Mean age: } 46.8 \\
\text { years }\end{array}$ & MBI-HSS & $\begin{array}{l}21.33 \\
(13.28)\end{array}$ & $\begin{array}{l}6.43 \\
(6.66)\end{array}$ & $35.78(8.94)$ & High EE: $49 \%$ & 7 \\
\hline $\begin{array}{l}\text { [28] } \\
\text { Chakraborty et al. } \\
2012 \\
\text { India }\end{array}$ & CS & $\begin{array}{l}\text { Psychiatric } \\
\text { nurses scoring } \\
<2 \text { on GHQ } \\
\text { From } 2 \\
\text { psychiatric } \\
\text { hospitals in } \\
\text { India }\end{array}$ & $100 \%$ & $\begin{array}{l}\mathrm{N}=101 \\
\text { Male: } 15.9 \% \\
\text { Female: } 84.1 \% \\
\text { Mean age: } 44 \\
\text { years }\end{array}$ & CBI & NR & NR & NR & NR & 6 \\
\hline $\begin{array}{l}\text { [29] } \\
\text { Coffey, } 1999 \\
\text { UK }\end{array}$ & CS & $\begin{array}{l}\text { Forensic } \\
\text { community } \\
\text { mental health } \\
\text { nurses in } \\
\text { England, Wales }\end{array}$ & $76.4 \%$ & $\begin{array}{l}\mathrm{N}=80 \\
\text { Male: } 53.8 \% \\
\text { Female: } 46.2 \% \\
\text { Mean age: } 37.8 \\
\text { years }\end{array}$ & MBI-HSS & $\begin{array}{l}19.3 \\
(10.1)\end{array}$ & $\begin{array}{l}5.65 \\
(4.3)\end{array}$ & $\begin{array}{l}33 \\
(6.2)\end{array}$ & $\begin{array}{l}\text { High EE: } 44.3 \% \\
\text { High DP: } 26.6 \% \\
\text { Low PA: } 26.6 \%\end{array}$ & 5 \\
\hline $\begin{array}{l}\text { [30] } \\
\text { Devilly et al } 2009 \\
\text { Australia }\end{array}$ & $\mathrm{CS}$ & $\begin{array}{l}\text { Mental health } \\
\text { professionals } \\
\text { working in }\end{array}$ & $31.7 \%$ & $\begin{array}{l}\mathrm{N}=152 \\
\text { Male: } 30 \% \\
\text { Female: } 70 \%\end{array}$ & CBI & NR & NR & NR & NR & 8 \\
\hline
\end{tabular}


Table 1 (Continued)

\begin{tabular}{|c|c|c|c|c|c|c|c|c|c|c|}
\hline Study & Design & $\begin{array}{l}\text { Study } \\
\text { population }\end{array}$ & $\begin{array}{l}\text { Response } \\
\text { rate }\end{array}$ & $\begin{array}{l}\text { Sample size } \\
\text { and } \\
\text { characteristics }\end{array}$ & $\begin{array}{l}\text { Burnout } \\
\text { measure }\end{array}$ & $\mathrm{EE}$ & $\mathrm{DP}$ & PA & $\begin{array}{l}\text { Burnout } \\
\text { Prevalence } \\
\text { High EE }>21 \\
\text { High DP }>8 \\
\text { Low PA }<28\end{array}$ & $\begin{array}{l}\text { Quality } \\
\text { Score } \\
\text { Good } \geq 8 \\
\text { Moderate } \\
6-7 \\
\text { Poor } \leq 5 \\
\end{array}$ \\
\hline & & $\begin{array}{l}\text { Victoria, } \\
\text { Australia }\end{array}$ & & $\begin{array}{l}\text { Psychologists } \\
(\mathrm{N}=125) \\
\text { Psychotherapists } \\
\text { Social workers ( } \\
\text { Psychiatrists }(\mathrm{N}= \\
\text { Nurse }(\mathrm{N}=1) \\
\text { Other }(\mathrm{N}=4)\end{array}$ & $\begin{array}{l}S(N=15) \\
N=6) \\
=1)\end{array}$ & & & & & \\
\hline $\begin{array}{l}\text { [31] } \\
\text { Edwards et al. } 2001 \\
\text { UK }\end{array}$ & CS & $\begin{array}{l}\text { Community } \\
\text { Mental Health } \\
\text { Nurses working } \\
\text { in Wales }\end{array}$ & $49 \%$ & $\begin{array}{l}\mathrm{N}=301 \\
\text { Male: } 38 \% \\
\text { Female: } 62 \% \\
\text { Mean age: } 40 \\
\text { years } \\
49 \% \text { had been in } \\
\text { current post >5 } \\
\text { years }\end{array}$ & MBI- HSS & $21.2(10.3)$ & $5.2(4.5)$ & $\begin{array}{l}34.8 \\
(6.5)\end{array}$ & $\begin{array}{l}\text { High EE: } 51 \% \\
\text { High DP: } 25 \% \\
\text { Low PA: } 14 \%\end{array}$ & 7 \\
\hline $\begin{array}{l}\text { [32] } \\
\text { Edwards et al. } 2006 \\
\text { UK }\end{array}$ & CS & $\begin{array}{l}\text { Community } \\
\text { Mental Health } \\
\text { Nurses working } \\
\text { in Wales }\end{array}$ & $32 \%$ & $\begin{array}{l}\mathrm{N}=260 \\
\text { Male: } 38 \% \\
\text { Female: } 62 \% \\
\text { Mean age: } 40 \\
\text { years } \\
\text { Mean length of } \\
\text { time working } \\
\text { as a CMHN was } \\
16 \text { years } \\
\text { Mean length of } \\
\text { time in current } \\
\text { job was } 6.5 \\
\text { years. }\end{array}$ & MBI- HSS & $\begin{array}{l}22.3 \\
(4.7)\end{array}$ & $\begin{array}{l}6.0 \\
(5.3)\end{array}$ & $\begin{array}{l}31.5 \\
(5.4)\end{array}$ & $\begin{array}{l}\text { High EE: } 36 \% \\
\text { High DP: } 12 \% \\
\text { Low PA: } 10 \%\end{array}$ & 6 \\
\hline $\begin{array}{l}\text { [33] } \\
\text { Evans et al. } 2006 \\
\text { UK }\end{array}$ & CS & $\begin{array}{l}\text { Mental Health } \\
\text { Social Workers } \\
\text { in UK }\end{array}$ & $39 \%$ & $\begin{array}{l}\mathrm{N}=237 \\
\text { Male: } 39 \% \\
\text { Female: } 61 \% \\
58 \% \text { of sample } \\
\text { were }<50 \text { years } \\
\text { old } \\
\text { Mean length of } \\
\text { time since } \\
\text { graduation was } \\
11.9 \text { years. }\end{array}$ & MBI-HSS & $26.3(10.1)$ & $7.3(5.2)$ & $33.9(6.8)$ & $\begin{array}{l}\text { Burnout } \\
\text { threshold: } 8 \% \\
\text { (Defined as all } \\
\text { three threshold } \\
E E>21 \text {, DP }>8 \text {, } \\
\text { PA }<28 \text { ) }\end{array}$ & 7 \\
\hline $\begin{array}{l}\text { [34] } \\
\text { Fong et al. } 2015 \\
\text { China }\end{array}$ & $\begin{array}{l}\text { LS } \\
2 \text { year } \\
\text { longitudinal } \\
\text { study } \\
7 \\
\text { measurement } \\
\text { times over } \\
2 \text { years }\end{array}$ & $\begin{array}{l}\text { Newly } \\
\text { employed } \\
\text { mental health } \\
\text { workers in } \\
\text { psychosocial } \\
\text { rehabilitation } \\
\text { institution in } \\
\text { Hong Kong } \\
50 \% \text { drop out } \\
\text { over } 2 \text { years }\end{array}$ & $89 \%$ & $\begin{array}{l}\mathrm{N}=312 \\
\text { Male: } 22.3 \% \\
\text { Female: } 77.7 \% \\
\text { Mean age: } 38.6 \\
\text { years }\end{array}$ & $\begin{array}{l}\text { CBI: work } \\
\text { sub-scale }\end{array}$ & NR & NR & NR & $\begin{array}{l}\text { Mean at } \\
\text { baseline: } 22 \\
\text { Increased over } \\
7 \text { time points. } \\
\text { Largest } \\
\text { increase from } \\
\text { year } 1 \text { - year } 2 \text {. } \\
2 \text { year follow } \\
\text { up mean score: } \\
34\end{array}$ & 6 \\
\hline $\begin{array}{l}\text { [35] } \\
\text { Garman et al. } 2002 \\
\text { USA }\end{array}$ & CS & $\begin{array}{l}\text { Staff and clients } \\
\text { from } 48 \\
\text { behavioural } \\
\text { health } \\
\text { programs in } \\
\text { mid western } \\
\text { USA. } \\
\text { Teams were } \\
\text { included only if } \\
\text { at least } 2 \text { clients } \\
\text { and } 2 \text { team } \\
\text { members } \\
\text { provided } \\
\text { completed } \\
\text { survey data } \\
31 \text { different } \\
\text { psychosocial } \\
\text { rehab teams } \\
\text { from } \\
\text { inpatient and } \\
\text { community } \\
\text { settings }\end{array}$ & $65 \%$ & $\begin{array}{l}\mathrm{N}=333 \\
\text { Male: } 25 \% \\
\text { Female: } 75 \% \\
\text { Teams from } \\
\text { public hospitals } \\
(\mathrm{N}=11) \\
\text { Community } \\
\text { based care } \\
\text { providers } \\
(\mathrm{N}=20)\end{array}$ & MBI-HSS & $17.2(10.9)$ & $5.4(5.2)$ & 37.4 (7.9) & NR & 5 \\
\hline
\end{tabular}




\begin{tabular}{|c|c|c|c|c|c|c|c|c|c|c|}
\hline $\begin{array}{l}\text { [36] } \\
\text { Galeazzi et al. } 2004 \\
\text { Italy }\end{array}$ & $\mathrm{CS}$ & $\begin{array}{l}30 \text { nurses, } 30 \\
\text { psychiatrists } \\
\text { working in } \\
\text { community } \\
\text { mental health } \\
\text { teams in } \\
\text { province of } \\
\text { Modena in Italy }\end{array}$ & $74 \%$ & $\begin{array}{l}\mathrm{N}=60 \\
\text { Male: } 32 \% \\
\text { Female: } 68 \%\end{array}$ & MBI-HSS & $\begin{array}{l}\text { Psych } \\
21.2(9.8) \\
\text { Nurses } 14.3 \\
(9.4)\end{array}$ & $\begin{array}{l}\text { Psych } \\
7.2(4) \\
\text { Nurses } \\
4.8(4.9)\end{array}$ & $\begin{array}{l}\text { Psych } 36.7 \\
(5.8) \\
\text { Nurses } 34.9 \\
(7.5)\end{array}$ & NR & 4 \\
\hline $\begin{array}{l}\text { [37] } \\
\text { Green et al. } 2013 \\
\text { USA }\end{array}$ & CS & $\begin{array}{l}\text { Community } \\
\text { mental health } \\
\text { staff working } \\
\text { with children } \\
\text { and families } \\
\text { from } 64 \\
\text { programs in } \\
\text { USA }\end{array}$ & $88.2 \%$ & $\begin{array}{l}\mathrm{N}=388 \\
\text { Male: } 24 \% \\
\text { Female: } 76 \% \\
\text { Mean age: } \\
35.74 \text { years old }\end{array}$ & $\begin{array}{l}\text { Emotional } \\
\text { exhaustion } \\
\text { subscale } \\
\text { from the } \\
\text { Children's } \\
\text { Services } \\
\text { Survey }\end{array}$ & NR & NR & NR & NR & 7 \\
\hline $\begin{array}{l}\text { [38] } \\
\text { Green et al. } 2014 \\
\text { USA }\end{array}$ & CS & $\begin{array}{l}\text { Clinical and } \\
\text { case } \\
\text { management } \\
\text { service } \\
\text { providers to } \\
\text { children, } \\
\text { adolescents } \\
\text { and families } \\
\text { within } 49 \\
\text { public-sector } \\
\text { programs in } \\
\text { San Diego, USA }\end{array}$ & $89 \%$ & $\begin{array}{l}\mathrm{N}=285 \\
\text { Male: } 18.6 \% \\
\text { Female: } 81.4 \% \\
\text { Mean age: } 36 \\
\text { years old }\end{array}$ & Organisatior & alNRocial Cont & extrscale & NR & NR & 8 \\
\hline $\begin{array}{l}\text { [39] } \\
\text { Hamaideh, } 2011 \\
\text { Jordan }\end{array}$ & CS & $\begin{array}{l}\text { All psychiatric } \\
\text { nurses in } \\
\text { Jordan }\end{array}$ & $82.3 \%$ & $\begin{array}{l}\mathrm{N}=181 \\
\text { Male: } 55.8 \% \\
\text { Female: } 44.2 \% \\
\text { Mean age: } \\
30.94 \text { years old }\end{array}$ & MBI-HSS & $\begin{array}{l}23.96 \\
(31.91)\end{array}$ & $\begin{array}{l}6.98 \\
(7.07)\end{array}$ & $\begin{array}{l}31.58 \\
(11.52)\end{array}$ & $\begin{array}{l}\text { High EE: } 54.7 \% \\
\text { High DP: } 34.2 \% \\
\text { Low PA: } 38.7 \%\end{array}$ & 6 \\
\hline $\begin{array}{l}\text { [40] } \\
\text { Happell et al. } 2003 \\
\text { Australia }\end{array}$ & CS & $\begin{array}{l}\text { Compare } \\
\text { forensic nurses } \\
\text { with } \\
\text { Community } \\
\text { nurses in } \\
\text { Melbourne, } \\
\text { Australia }\end{array}$ & $67.5 \%$ & $\begin{array}{l}\mathrm{N}=129 \\
\text { Forensic nurses } \\
\mathrm{N}=51 \\
\text { Community } \\
\text { nurses } \mathrm{N}=78\end{array}$ & MBI-HSS & $\begin{array}{l}\text { Forensic } \\
12.9 \\
(7.5) \\
\text { CMHT } \\
17.4 \\
(12.2)\end{array}$ & $\begin{array}{l}\text { Forensic } \\
4.7 \\
(6) \\
\text { CMHT } \\
4.5 \\
(4.9)\end{array}$ & $\begin{array}{l}\text { Forensic } \\
34.5 \\
(7.9) \\
\text { CMHT } \\
35.6 \\
(9.8)\end{array}$ & $\begin{array}{l}\text { Forensic } \\
\text { High EE: } 15.6 \% \\
\text { High DP: } 17.6 \% \\
\text { Low PA: } 17.6 \% \\
\text { CMHT } \\
\text { High EE } 35.8 \% \\
\text { High DP: } 24.3 \% \\
\text { Low PA: } 23 \%\end{array}$ & 5 \\
\hline $\begin{array}{l}\text { [41] } \\
\text { Imai et al. } 2006 \\
\text { Japan }\end{array}$ & CS & $\begin{array}{l}\text { Public Health } \\
\text { Nurses } \\
396 \text { psychiatry } \\
389 \text { non } \\
\text { psychiatry }\end{array}$ & $74.7 \%$ & $\begin{array}{l}\mathrm{N}=785 \\
\text { Mean age: } 41.4 \\
\text { years old } \\
\text { Mean length of } \\
\text { career as PHN } \\
18 \text { years } \\
\text { Mean length of } \\
\text { time in current } \\
\text { service was } 3 \\
\text { years. }\end{array}$ & $\begin{array}{l}\text { Pines } \\
\text { Burnout } \\
\text { Measure }\end{array}$ & NR & NR & NR & NR & 7 \\
\hline $\begin{array}{l}\text { [42] } \\
\text { Imai et al. } 2004 \\
\text { Japan }\end{array}$ & $\mathrm{CS}$ & $\begin{array}{l}\text { Public health } \\
\text { nurses who } \\
\text { work in mental } \\
\text { health } \\
\text { compared with } \\
\text { general public } \\
\text { mental health } \\
\text { nurses }\end{array}$ & $80.6 \%$ & $\begin{array}{l}\mathrm{N}=396 \\
\text { Mean length of } \\
\text { career as PHN } \\
19 \text { years }\end{array}$ & $\begin{array}{l}\text { Pines } \\
\text { Burnout } \\
\text { Measure }\end{array}$ & NR & NR & NR & $\begin{array}{l}\text { Burnout: } 59.2 \% \\
\text { Defined as } \\
\text { mean response } \\
\text { of }>3 \text { for all } \\
\text { items. }\end{array}$ & 7 \\
\hline $\begin{array}{l}\text { [43] } \\
\text { Jahrami } 2009 \\
\text { Bahrain }\end{array}$ & CS & $\begin{array}{l}\text { Psychiatric } \\
\text { nurses, doctors, } \\
\text { occupational } \\
\text { therapists, } \\
\text { social workers, } \\
\text { psychologists }\end{array}$ & $58 \%$ & $N=153$ & MBI-HSS & $\begin{array}{l}18.96 \\
(13.81)\end{array}$ & $\begin{array}{l}6.69 \\
(5.26)\end{array}$ & 34.28 & NR & 5 \\
\hline $\begin{array}{l}\text { [44] } \\
\text { Jeanneau and } \\
\text { Armelius } \\
2000 \\
\text { Sweden }\end{array}$ & $\mathrm{CS}$ & $\begin{array}{l}\text { MHPs working } \\
\text { in a variety of } \\
\text { settings across } \\
\text { Sweden: } \\
\text { psychiatric } \\
\text { wards, small } \\
\text { psychiatric } \\
\text { treatment } \\
\text { homes, forensic } \\
\text { wards, } \\
\text { community } \\
\text { care settings }\end{array}$ & $100 \%$ & $\begin{array}{l}\mathrm{N}=754 \\
\text { Male: } 39 \% \\
\text { Female: } 61 \% \\
\text { Mean age: } 45 \\
\text { Psychiatric aids } \\
(\mathrm{N}=430) \\
\text { Nurses } \\
(\mathrm{N}=113)\end{array}$ & $\begin{array}{l}\text { MBI-HSS } \\
\text { Pines } \\
\text { Burnout } \\
\text { Measure }\end{array}$ & NR & NR & NR & NR & 7 \\
\hline $\begin{array}{l}\text { [45] } \\
\text { Johnson et al., } 2012 \\
\text { UK }\end{array}$ & CS & $\begin{array}{l}\text { MHPs from } 100 \\
\text { wards \& } 36\end{array}$ & $64 \%$ & $\begin{array}{l}\mathrm{N}=2258 \\
\text { Male: } 36 \% \\
\text { Female: } 64 \%\end{array}$ & MBI-GS & $\begin{array}{l}\frac{\mathbf{E x}}{20}_{1} \\
(12)\end{array}$ & $\frac{C y}{N R}$ & $\begin{array}{l}\frac{\text { PA }}{33.7}_{(8.3)}\end{array}$ & $\begin{array}{l}\text { High Ex: } 49 \% \text { of } \\
\text { staff in acute } \\
\text { general wards }\end{array}$ & 8 \\
\hline
\end{tabular}


Table 1 (Continued)

\begin{tabular}{|c|c|c|c|c|c|c|c|c|c|c|}
\hline Study & Design & $\begin{array}{l}\text { Study } \\
\text { population }\end{array}$ & $\begin{array}{l}\text { Response } \\
\text { rate }\end{array}$ & $\begin{array}{l}\text { Sample size } \\
\text { and } \\
\text { characteristics }\end{array}$ & $\begin{array}{l}\text { Burnout } \\
\text { measure }\end{array}$ & $\mathrm{EE}$ & $\mathrm{DP}$ & PA & $\begin{array}{l}\text { Burnout } \\
\text { Prevalence } \\
\text { High EE }>21 \\
\text { High DP }>8 \\
\text { Low PA }<28\end{array}$ & $\begin{array}{l}\text { Quality } \\
\text { Score } \\
\text { Good } \geq 8 \\
\text { Moderate } \\
6-7 \\
\text { Poor } \leq 5\end{array}$ \\
\hline & & $\begin{array}{l}\text { CMHTs in } \\
\text { England }\end{array}$ & & $\begin{array}{l}\text { Nurses } \\
(\mathrm{N}=1054) \\
\text { Doctors } \\
(\mathrm{N}=135) \\
\text { Psychologists } \\
(\mathrm{N}=44) \\
\text { Occupational } \\
\text { Therapists } \\
(\mathrm{N}=82) \\
\text { Nursing } \\
\text { assistants/ } \\
\text { support } \\
\text { workers } \\
(\mathrm{N}=640) \\
\text { Social workers } \\
\text { ( } \mathrm{N}=86) \\
\text { Ward managers } \\
(\mathrm{N}=111)\end{array}$ & & & & & 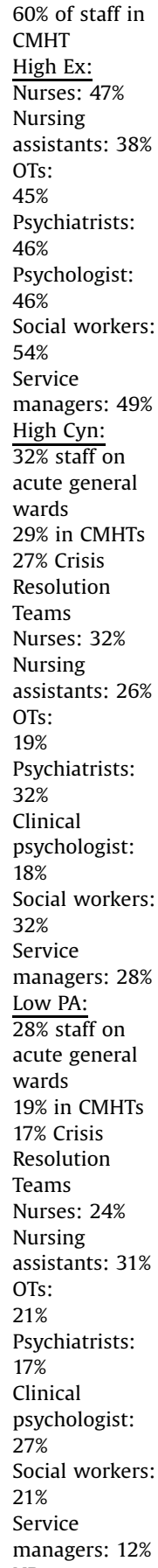 & \\
\hline $\begin{array}{l}\text { [46] } \\
\text { Johnson et al. } 2016 \\
\text { UK }\end{array}$ & $\mathrm{CS}$ & $\begin{array}{l}\text { One } \\
\text { independent } \\
\text { forensic } \\
\text { psychiatric } \\
\text { hospital group, } \\
2 \text { sites. }\end{array}$ & $97 \%$ & $\begin{array}{l}\mathrm{N}=114 \\
\text { Male: } 42 \% \\
\text { Female: } 78 \% \\
\text { Staff based in: } \\
\text { Medium secure } \\
\text { unit }(\mathrm{N}=47) \\
\text { Low secure unit } \\
(\mathrm{N}=50) \\
\text { Medium/low } \\
\text { secure }(\mathrm{N}=6) \\
\text { Locked } \\
\text { rehabilitation }\end{array}$ & MBI-HSS & NR & NR & NR & NR & 7 \\
\hline
\end{tabular}




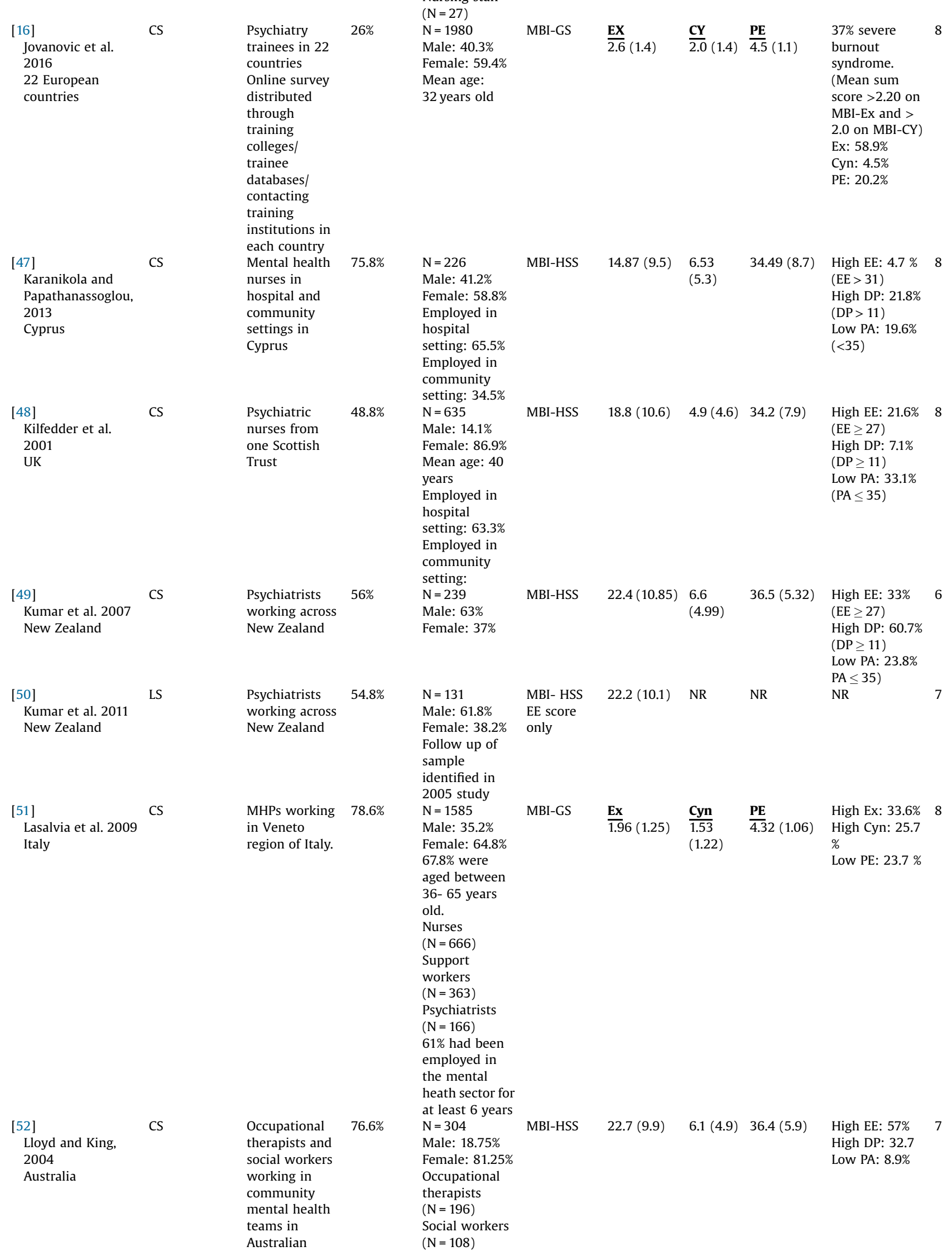

$(\mathrm{N}=11)$

Support

workers

$(\mathrm{N}=66)$

Nursing staff

Mean age:

Employed in

hospital

Employed in

community

setting: $34.5 \%$

Female: $86.9 \%$

Mean age: 40

setting: $63.3 \%$

Employed in

community

Male: 63\%

working across

$(4.99)$

High DP: $60.7 \%$

DP $\geq 11)$

$\mathrm{PA} \leq 35)$

Male: $61.8 \%$

Female: $38.2 \%$

sample

identified in

2005 study

$\mathrm{N}=1585$

Female: $64.8 \%$

$67.8 \%$ were

old.

$(\mathrm{N}=363)$

Psychiatrists

$(\mathrm{N}=166)$

$61 \%$ had been

employed in

the mental

Male: $18.75 \%$

Female: $81.25 \%$

$(\mathrm{N}=108)$ 
Table 1 (Continued)

\begin{tabular}{|c|c|c|c|c|c|c|c|c|c|c|}
\hline Study & Design & $\begin{array}{l}\text { Study } \\
\text { population }\end{array}$ & $\begin{array}{l}\text { Response } \\
\text { rate }\end{array}$ & $\begin{array}{l}\text { Sample size } \\
\text { and } \\
\text { characteristics }\end{array}$ & $\begin{array}{l}\text { Burnout } \\
\text { measure }\end{array}$ & $\mathrm{EE}$ & DP & PA & $\begin{array}{l}\text { Burnout } \\
\text { Prevalence } \\
\text { High EE }>21 \\
\text { High DP }>8 \\
\text { Low PA }<28\end{array}$ & $\begin{array}{l}\text { Quality } \\
\text { Score } \\
\text { Good } \geq 8 \\
\text { Moderate } \\
6-7 \\
\text { Poor } \leq 5 \\
\end{array}$ \\
\hline $\begin{array}{l}\text { [53] } \\
\text { Levert et al. } 2000 \\
\text { South Africa }\end{array}$ & CS & $\begin{array}{l}\text { public health } \\
\text { system } \\
\text { Psychiatric } \\
\text { nurses working } \\
\text { in psychiatric } \\
\text { and general } \\
\text { hospitals in } \\
\text { South Africa }\end{array}$ & $27 \%$ & $N=94$ & MBI-HSS & $29.9(12.93)$ & $\begin{array}{l}9.63 \\
(4.63)\end{array}$ & $19.16(8.26)$ & $\begin{array}{l}\text { High EE: } 54.9 \% \\
\text { High DP: } 45 \% \\
\text { Low PA: } 3.3 \%\end{array}$ & 6 \\
\hline $\begin{array}{l}\text { [54] } \\
\text { Madathil et al. } \\
2014 \\
\text { USA }\end{array}$ & CS & $\begin{array}{l}\text { Psychiatric } \\
\text { nurses working } \\
\text { in } 2 \text { hospital } \\
\text { groups in } \\
\text { Montana and } \\
\text { New York }\end{array}$ & & $\begin{array}{l}\mathrm{N}=89 \\
\text { Male: } 12 \% \\
\text { Female: } 88 \% \\
33 \%(\mathrm{n}=29) \\
\text { Montana State } \\
\text { Hospital } \\
67 \%(\mathrm{n}=60) \\
\text { New York State } \\
\text { hospitals }\end{array}$ & MBI-HSS & $\begin{array}{l}31.3 \\
(12)\end{array}$ & $\begin{array}{l}12.2 \\
(5)\end{array}$ & $\begin{array}{l}43.84 \\
(7.8)\end{array}$ & NR & 8 \\
\hline $\begin{array}{l}\text { [55] } \\
\text { Melchior et al. } 1997 \\
\text { Netherlands }\end{array}$ & $\mathrm{CS}$ & $\begin{array}{l}\text { Nurses working } \\
\text { in long stay } \\
\text { psychiatric } \\
\text { wards in five } \\
\text { psychiatric } \\
\text { hospital in } \\
\text { Netherlands }\end{array}$ & $73.4 \%$ & $\begin{array}{l}\mathrm{N}=361 \\
\text { Male: } 28 \% \\
\text { Female: } 72 \% \\
\text { Mean age: } 35 \\
\text { years } \\
\text { Respondents } \\
\text { working in } \\
\text { psychiatry for } \\
\text { an average of } \\
13.5 \text { years }\end{array}$ & MBI- HSS & $17.22(7.67)$ & $\begin{array}{l}6.51 \\
(4.02)\end{array}$ & $31.97(4.14)$ & NR & 7 \\
\hline $\begin{array}{l}\text { [56] } \\
\text { Ndetei et al. } 2008 \\
\text { Kenya }\end{array}$ & CS & $\begin{array}{l}\text { MHP working } \\
\text { in Mathari } \\
\text { Psychiatric } \\
\text { hospital. Only } \\
\text { national } \\
\text { referral and } \\
\text { teaching } \\
\text { psychiatric } \\
\text { hospital in } \\
\text { Kenya. } 600 \text { in } \\
\text { patient beds. }\end{array}$ & $71.6 \%$ & $\begin{array}{l}\mathrm{N}=285 \\
\text { Male: } 35.5 \% \\
\text { Female: } 64.5 \% \\
\text { Clinical staff } \\
\text { (doctors, } \\
\text { nurses, } \\
\text { pharmacists) } \\
(\mathrm{N}=80) \\
\text { Non clinical } \\
\text { staff }(\mathrm{N}=14) \\
\text { Support staff } \\
(\mathrm{N}=27)\end{array}$ & MBI- HSS & $17.2(9.78)$ & $7.3(6.5)$ & $29.3(10.26)$ & $\begin{array}{l}\text { High EE: } 38 \% \\
\text { High DP: } 47.8 \% \\
\text { Low PA: } 38.6 \% \\
\text { Cut off point on } \\
\text { scale unclear }\end{array}$ & 7 \\
\hline $\begin{array}{l}\text { [57] Nelson et al. } \\
2009 \\
\text { UK }\end{array}$ & CS & $\begin{array}{l}\text { MHPs working } \\
\text { in Crisis } \\
\text { Resolution } \\
\text { Teams (CRT), } \\
\text { Assertive } \\
\text { Outreach } \\
\text { Teams (AOT), } \\
\text { Community } \\
\text { Mental Health } \\
\text { Teams (CMHT) } \\
\text { in UK }\end{array}$ & $78 \%$ & $\begin{array}{l}\mathrm{N}=433 \\
\text { Male: } 47.3 \% \\
\text { Female: } 52.7 \% \\
\text { Nursing } \\
(\mathrm{N}=196) \\
\text { Social work } \\
(\mathrm{N}=82) \\
\text { OT }(\mathrm{N}=23) \\
\text { Psychiatry } \\
(\mathrm{N}=48) \\
\text { CRT }(\mathrm{N}=132) \\
\text { AOT }(\mathrm{N}=187) \\
\text { CMHT }(\mathrm{N}=114)\end{array}$ & MBI-HSS & $\begin{array}{l}\text { CRT } \\
17.7 \\
(\mathrm{NR}) \\
\text { AOT } \\
17.4 \\
(\mathrm{NR}) \\
\text { CMHT } \\
19 \\
(\mathrm{NR})\end{array}$ & $\begin{array}{l}\text { CRT } \\
4.8 \\
\text { (NR) } \\
\text { AOT } \\
4.4 \\
\text { (NR) } \\
\text { CMHT } \\
5.7 \\
(\mathrm{NR})\end{array}$ & $\begin{array}{l}\text { CRT } \\
36.7 \\
(\mathrm{NR}) \\
\text { AOT } \\
34.8 \\
\text { (NR) } \\
\text { CMHT } \\
32.7 \\
(\mathrm{NR})\end{array}$ & NR & 7 \\
\hline $\begin{array}{l}\text { [58] } \\
\text { Oddie and Ousley, } \\
2007 \\
\text { UK }\end{array}$ & CS & $\begin{array}{l}\text { Nurses and } \\
\text { occupational } \\
\text { therapists from } \\
\text { three wards in } \\
\text { one medium } \\
\text { secure } \\
\text { psychiatric } \\
\text { hospital in UK }\end{array}$ & $57.3 \%$ & $\begin{array}{l}\mathrm{N}=71 \\
\text { Male: } 60 \% \\
\text { Female: } 40 \% \\
\text { Mean age } 34 \\
\text { years old } \\
\text { Mean length of } \\
\text { time } \\
\text { respondents } \\
\text { reported } \\
\text { working in } \\
\text { current } \\
\text { location was } \\
4.8 \text { years }\end{array}$ & MBI-HSS & 23 (10.9) & $7(6.7)$ & $35(8.2)$ & $\begin{array}{l}\text { High EE: } 54 \% \\
\text { High DP: } 35 \% \\
\text { Low PA: } 15 \%\end{array}$ & 7 \\
\hline $\begin{array}{l}\text { [59] } \\
\text { Ogresta et al. } 2008 \\
\text { Croatia }\end{array}$ & CS & $\begin{array}{l}3 \text { state } \\
\text { hospitals and } \\
12 \text { clinics in } \\
\text { Croatia }\end{array}$ & $50 \%$ & $\begin{array}{l}\mathrm{N}=174 \\
\text { Male: } 20.1 \% \\
\text { Female: } 79.9 \% \\
\text { Mean age } \\
41.2 \text { years old } \\
\text { Nurses }(\mathrm{N}=86) \\
\text { Social Workers }\end{array}$ & MBI-HSS & $24.5(9.2)$ & $\begin{array}{l}16.6 \\
(7.6)\end{array}$ & $\begin{array}{l}21.8 \\
(7.4)\end{array}$ & NR & 7 \\
\hline
\end{tabular}




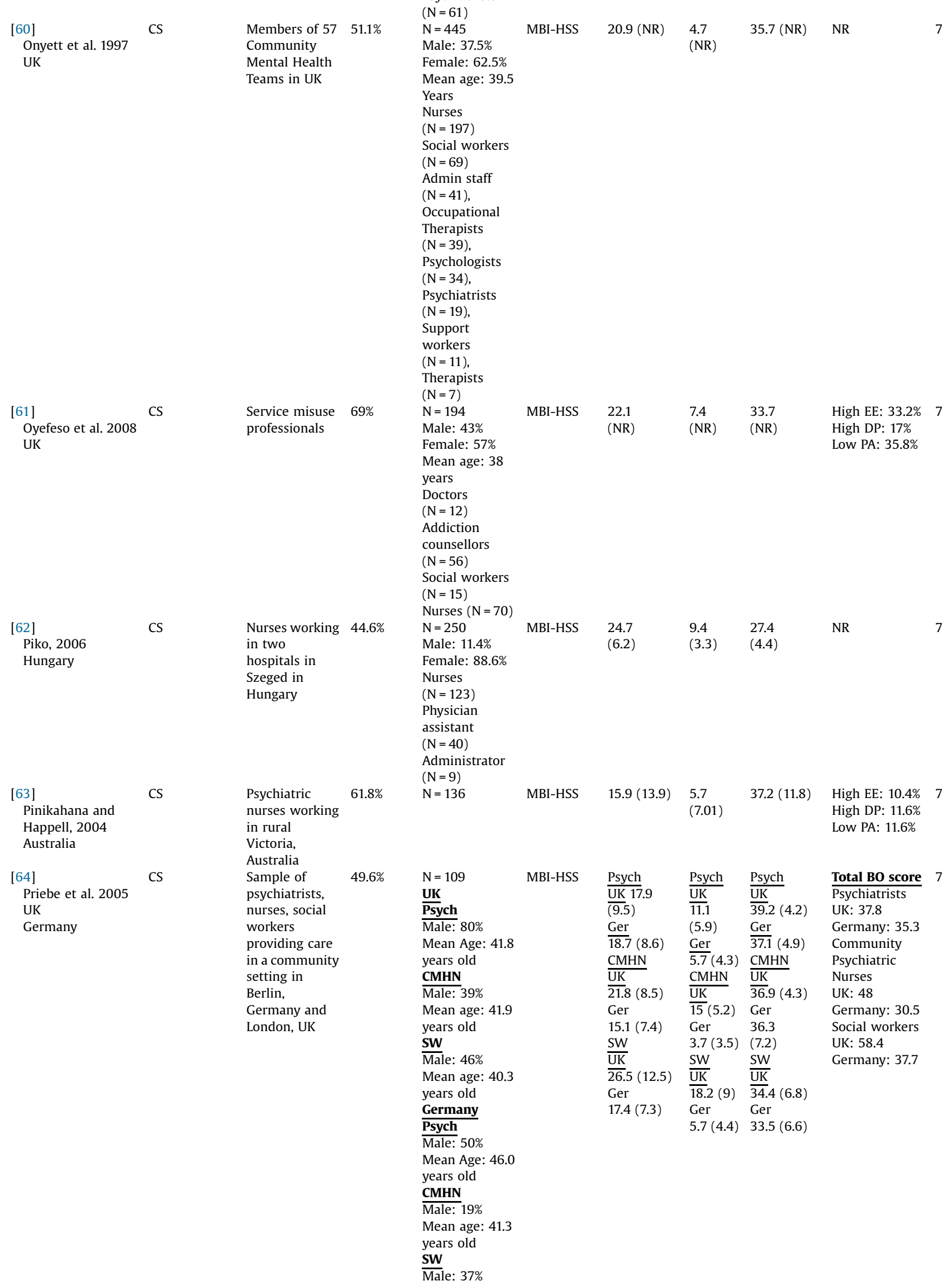

$(\mathrm{N}=27)$

Psychiatrist

$(\mathrm{N}=61)$

Male: $37.5 \%$

emale: $62.5 \%$

$(\mathrm{N}=197)$

Social workers

$\mathrm{N}=69$ )

$(\mathrm{N}=41)$

Occupational

Psychologists

workers

$\mathrm{N}=194$

Male: 43\%

years

counsellors

$(\mathrm{N}=56)$

$(\mathrm{N}=15)$

Nurses $(\mathrm{N}=70)$

Nurses

$(\mathrm{N}=123)$

$(\mathrm{N}=9)$

nurses working

in rural

Sample of

Germany and

ondon, UK 
Table 1 (Continued)

\begin{tabular}{|c|c|c|c|c|c|c|c|c|c|c|}
\hline Study & Design & $\begin{array}{l}\text { Study } \\
\text { population }\end{array}$ & $\begin{array}{l}\text { Response } \\
\text { rate }\end{array}$ & $\begin{array}{l}\text { Sample size } \\
\text { and } \\
\text { characteristics }\end{array}$ & $\begin{array}{l}\text { Burnout } \\
\text { measure }\end{array}$ & $\mathrm{EE}$ & DP & PA & $\begin{array}{l}\text { Burnout } \\
\text { Prevalence } \\
\text { High EE }>21 \\
\text { High DP }>8 \\
\text { Low PA }<28\end{array}$ & $\begin{array}{l}\text { Quality } \\
\text { Score } \\
\text { Good } \geq 8 \\
\text { Moderate } \\
6-7 \\
\text { Poor } \leq 5 \\
\end{array}$ \\
\hline $\begin{array}{l}\text { [65] } \\
\text { Prosser et al. } 1997 \\
\text { UK }\end{array}$ & CS & $\begin{array}{l}\text { MHPs from } 3 \\
\text { mental health } \\
\text { sectors in inner } \\
\text { city London. } \\
\text { Included staff } \\
\text { working in in- } \\
\text { patient and } \\
\text { out-patient } \\
\text { settings. }\end{array}$ & $76 \%$ & $\begin{array}{l}\text { Mean age: } 40.6 \\
\text { years old } \\
\mathrm{N}=121 \\
\text { Male: } 43 \% \\
\text { Female: } 57 \% \\
\text { Nurses: } 71 \\
\text { Psychiatrists: } \\
23 \\
66 \% \text { of staff had } \\
\text { been in current } \\
\text { job for less than } \\
2 \text { years. }\end{array}$ & MBI- HSS & NR & NR & NR & NR & 7 \\
\hline (1) & 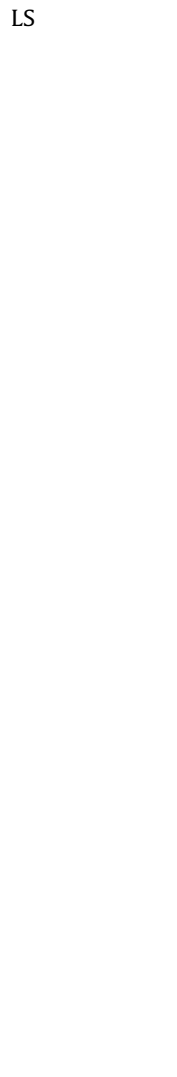 & $\begin{array}{l}\text { Study } \\
\text { completed } \\
\text { during move } \\
\text { from } \\
\text { institutional } \\
\text { setting to } \\
\text { community } \\
\text { based model }\end{array}$ & $\begin{array}{l}\mathbf{1 9 9 4} 76 \% \\
\mathbf{1 9 9 5} \\
60 \% \\
\mathbf{1 9 9 6} \\
62 \%\end{array}$ & $\begin{array}{l}1994 \\
\mathrm{~N}=120 \\
\text { Nurses: } \mathrm{N}=80 \\
\text { Psychiatrists: } \\
\mathrm{N}=23 \\
\text { In-patient: } \\
\mathrm{N}=50 \\
\text { Community: } \\
\mathrm{N}=29 \\
\text { Day/outpatient } \\
\text { in main } \\
\text { hospital: } \mathrm{N}=42 \\
\text { 1995 } \\
\mathrm{N}=166 \\
\text { Nurses: } \mathrm{N}=63 \\
\text { Psychiatrists: } \\
\mathrm{N}=17 \\
\text { In-patient: } \\
\mathrm{N}=35 \\
\text { Community: } \\
\mathrm{N}=65 \\
\text { Day/outpatient } \\
\text { in main } \\
\text { hospital: } \mathrm{N}=0 \\
\text { 1996 } \\
\mathrm{N}=94 \\
\text { Nurses: } \mathrm{N}=64 \\
\text { Psychiatrists: } \\
\mathrm{N}=12 \\
\text { In-patient: } \\
\mathrm{N}=35 \\
\text { Community: } \\
\mathrm{N}=59 \\
\text { Day/outpatient } \\
\text { in main } \\
\text { hospital: } \mathrm{N}=0\end{array}$ & 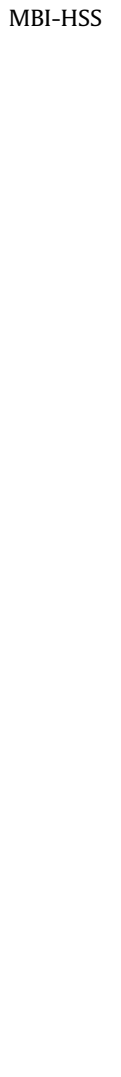 & $\begin{array}{l}\text { ‘94 } \\
\mathrm{EE}: 22.9 \\
(11.1) \\
\mathbf{9 5} \\
\mathrm{EE}: 24.3 \\
(11.2) \\
\mathbf{9 6} \\
\mathrm{EE}: \\
21.1(9.7)\end{array}$ & $\begin{array}{l}\mathbf{9 9 4} \\
\text { DP: } 7.5 \\
(5.7) \\
\text { ‘95 } \\
\text { DP: } \\
8.1 \\
(6.1) \\
\text { '96 } \\
\text { DP: } \\
7.5(5.5)\end{array}$ & $\begin{array}{l}\mathbf{9 4} \\
\text { PA: } 33.5 \\
(6.6) \\
\mathbf{9 5} \\
\text { PA: } \\
33 \\
(6.5) \\
\text { '96} \\
\text { PA: } \\
34.2(6.2)\end{array}$ & NR & 7 \\
\hline $\begin{array}{l}\text { [67] } \\
\text { Rogala et al. } 2015 \\
\text { USA, Poland } \\
\text { \& } \\
\text { [68] } \\
\text { Shoji et al. } 2015 \\
\text { USA, Poland }\end{array}$ & $\begin{array}{l}\text { LS } \\
\text { Baseline (T1) } \\
\text { and } 6 \text { month } \\
\text { follow up (T2) }\end{array}$ & & & $\begin{array}{l}\text { Study 1 } \\
\text { T1: } 294 \\
\text { T2: } 135 \\
\text { MHPs in USA } \\
\text { military } \\
\text { working with } \\
\text { people } \\
\text { suffering from } \\
\text { trauma } \\
\text { Mean age: } \\
\text { T1 } 48.87 \text { years } \\
\text { T2 } 50.62 \text { years } \\
\text { Study } 2 \\
\text { T1: } 306 \\
\text { T2: } 193 \\
\text { Health } \\
\text { providers, } \\
\text { social workers } \\
\text { working with } \\
\text { civilian trauma } \\
\text { survivors in } \\
\text { Poland. } \\
\text { Mean age: } \\
\text { T1:35.32 years }\end{array}$ & OLBI & NR & NR & NR & NR & 7 \\
\hline
\end{tabular}




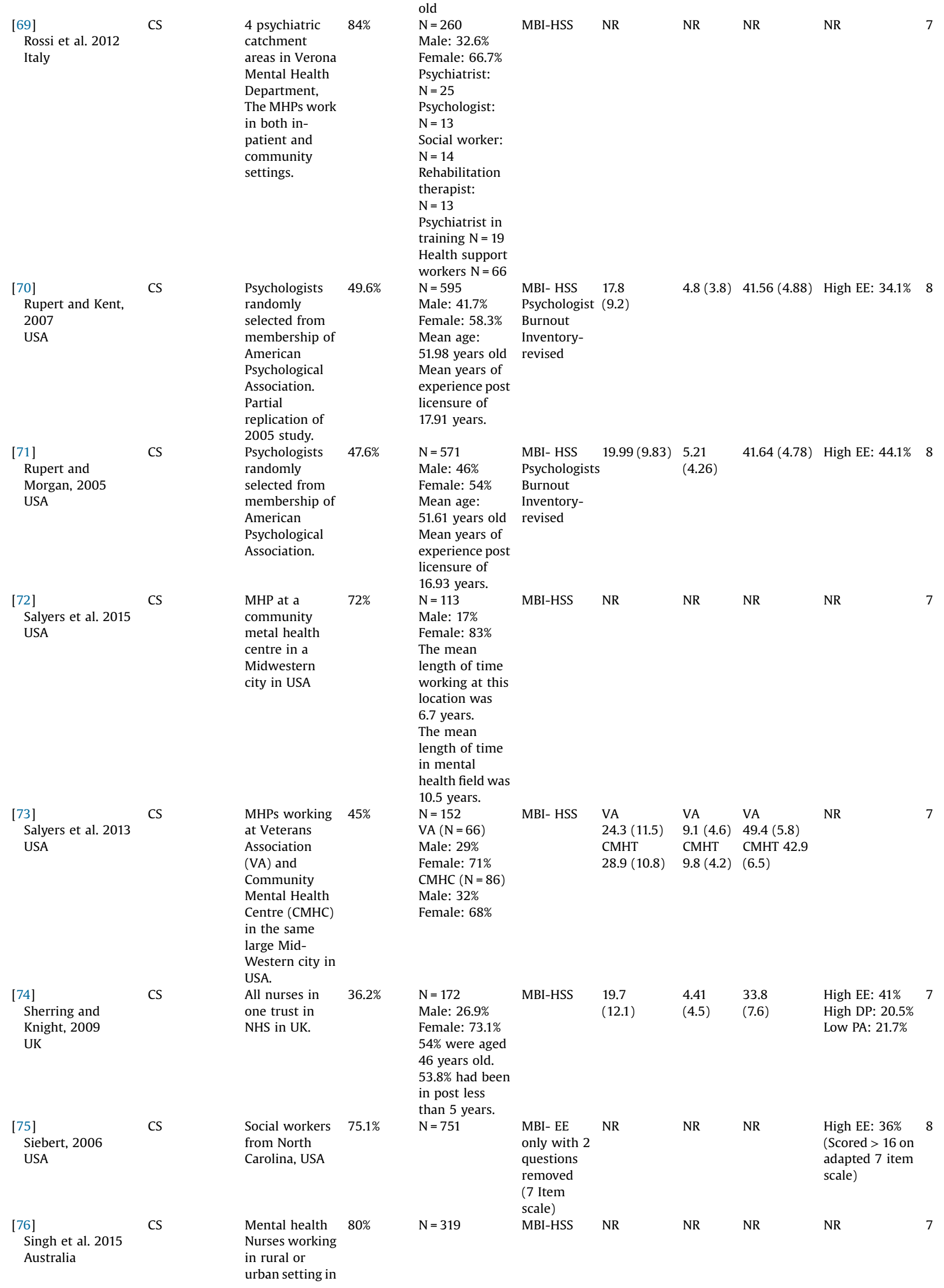


Table 1 (Continued)

\begin{tabular}{|c|c|c|c|c|c|c|c|c|c|c|}
\hline Study & Design & $\begin{array}{l}\text { Study } \\
\text { population }\end{array}$ & $\begin{array}{l}\text { Response } \\
\text { rate }\end{array}$ & $\begin{array}{l}\text { Sample size } \\
\text { and } \\
\text { characteristics }\end{array}$ & $\begin{array}{l}\text { Burnout } \\
\text { measure }\end{array}$ & $\mathrm{EE}$ & DP & PA & $\begin{array}{l}\text { Burnout } \\
\text { Prevalence } \\
\text { High EE }>21 \\
\text { High DP }>8 \\
\text { Low PA }<28\end{array}$ & $\begin{array}{l}\text { Quality } \\
\text { Score } \\
\text { Good } \geq 8 \\
\text { Moderate } \\
6-7 \\
\text { Poor } \leq 5\end{array}$ \\
\hline $\begin{array}{l}\text { [77] } \\
\text { Sorgaard et al. } \\
2007 \\
5 \text { European } \\
\text { countries: } \\
\text { Denmark } \\
\text { Norway } \\
\text { UK } \\
\text { Finland } \\
\text { Poland }\end{array}$ & $\begin{array}{l}\text { LS } \\
\text { Baseline } \\
6 \text { months } \\
12 \text { months }\end{array}$ & $\begin{array}{l}\text { four states in } \\
\text { Australia } \\
\text { (Victoria, New } \\
\text { South Wales, } \\
\text { Queensland } \\
\text { and Western } \\
\text { Australia) } \\
\text { In patient and } \\
\text { community } \\
\text { MHP in } 6 \\
\text { psychiatric } \\
\text { centres in } 5 \\
\text { European } \\
\text { countries }\end{array}$ & $72 \%$ & $\begin{array}{l}\mathrm{N}=414 \\
\text { In patient staff: } \\
\mathrm{N}=205 \\
\text { Male: } 15.3 \% \\
\text { Female: } 84.7 \% \\
\text { Mean age: } \\
40.2 \text { years } \\
\text { Community } \\
\text { staff: } \mathrm{N}=209 \\
\text { Male: } 16.3 \% \\
\text { Female: } 83.7 \% \\
\text { Mean age: } 43.9 \\
\text { years }\end{array}$ & MBI-HSS & $\begin{array}{l}\text { In patient } \\
15.8 \\
(9.7) \\
\text { Community } \\
18.3 \\
(10.5)\end{array}$ & $\begin{array}{l}\text { In } \\
\text { patient } \\
4.9 \\
(4.7) \\
\text { Comm } \\
\text { unity } \\
4.5 \\
(4.4)\end{array}$ & $\begin{array}{l}\text { In patient } \\
36.3 \\
(8.1) \\
\text { Community } \\
36.4(7.4)\end{array}$ & BO: $1.2 \%$ & 8 \\
\hline $\begin{array}{l}\text { [78] } \\
\text { Sorgaard et al. } 2010 \\
5 \text { European } \\
\text { countries: } \\
\text { Denmark } \\
\text { Norway } \\
\text { UK } \\
\text { Finland } \\
\text { Poland }\end{array}$ & CS & $\begin{array}{l}\text { Qualified and } \\
\text { unqualified } \\
\text { mental health } \\
\text { nursing staff }\end{array}$ & $72 \%$ & $\begin{array}{l}\mathrm{N}=196 \\
\text { Qualified } \\
\mathrm{N}=124 \\
\text { Male: } 16.9 \% \\
\text { Female: } 84.1 \% \\
\text { Mean age: } 40.8 \\
\text { years } \\
\text { Unqualified } \\
\mathrm{N}=72 \\
\text { Male: } 27.8 \% \\
\text { Female: } 72.2 \% \\
\text { Mean age: } 43.1 \\
\text { years old }\end{array}$ & MBI-HSS & $\begin{array}{l}15.6 \\
(8.9)\end{array}$ & $3.6(4.7)$ & $\begin{array}{l}36.3 \\
(7.4)\end{array}$ & $\begin{array}{l}\text { High EE: } 8.9 \% \\
\text { High DP: } 8.0 \% \\
\text { Low PA: } 25.4 \%\end{array}$ & 7 \\
\hline $\begin{array}{l}\text { [79] } \\
\text { Spear et al. } 2004 \\
\text { Australia }\end{array}$ & CS & $\begin{array}{l}\text { MHPs working } \\
\text { in eight } \\
\text { metropolitan } \\
\text { mental health } \\
\text { services for } \\
\text { older adults in } \\
\text { Western } \\
\text { Australia. }\end{array}$ & $33 \%$ & $\begin{array}{l}\mathrm{N}=116 \\
\text { Psychiatrists } \\
\mathrm{N}=13 \\
\text { Nurses } \mathrm{N}=66 \\
\text { AHP } \mathrm{N}=18 \\
\text { Admins staff } \\
\mathrm{N}=5 \\
\text { Managers } \mathrm{N}=4 \\
\text { Other } \mathrm{N}=10\end{array}$ & MBI-HSS & NR & NR & NR & NR & 7 \\
\hline $\begin{array}{l}\text { [80] } \\
\text { Steel et al. } 2015 \\
\text { UK }\end{array}$ & CS & $\begin{array}{l}\text { Psychotherapists } \\
\text { working AT eight } \\
\text { Improving access } \\
\text { Psychological Th } \\
\text { services in UK }\end{array}$ & $\begin{array}{l}44.3 \% \\
\text { s to } \\
\text { erapy }\end{array}$ & $\begin{array}{l}\mathrm{N}=116 \\
\text { Male: } 21 \% \\
\text { Female: } 79 \% \\
\text { Mean age: } 36.9 \\
\text { years old } \\
\text { Mean length of } \\
\text { years in } \\
\text { practice was } 1.9 \\
\text { years }\end{array}$ & MBI-HSS & $\begin{array}{l}20.47(9.7) \\
0\end{array}$ & $\begin{array}{l}3.26 \\
(3.45)\end{array}$ & $38.71(5.36)$ & NR & 7 \\
\hline $\begin{array}{l}\text { [81] } \\
\text { Tummers et al. } \\
2001 \\
\text { Netherlands }\end{array}$ & CS & $\begin{array}{l}\text { Psychiatric } \\
\text { nurses from } 5 \\
\text { different wards } \\
\text { in one } \\
\text { psychiatric } \\
\text { hospital in the } \\
\text { Netherlands }\end{array}$ & $63.6 \%$ & $\begin{array}{l}\mathrm{N}=178 \\
\text { Nurses: } \mathrm{N}=151 \\
\text { Male: } 47 \% \\
\text { Female: } 53 \% \\
\text { Mean age: } 34 \\
\text { years old }\end{array}$ & MBI-GS EE & NR & NR & NR & NR & 7 \\
\hline $\begin{array}{l}\text { [82] } \\
\text { Volpe et al. } 2014 \\
\text { Italy }\end{array}$ & $\mathrm{CS}$ & $\begin{array}{l}\text { Early career } \\
\text { MHPs (defined } \\
\text { as those who } \\
\text { completed } \\
\text { their training in } \\
\text { psychiatry } \\
\text { within } 5 \text { years } \\
\text { and/or were } \\
\text { below the age } \\
\text { of } 40 \text { years old) }\end{array}$ & $71.4 \%$ & $\begin{array}{l}\mathrm{N}=100 \\
\text { Psychiatrists } \\
\mathrm{N}=50, \\
\text { Non medical } \\
\text { MHPs } \mathrm{N}=50 \\
\text { Male: } 52 \% \\
\text { Female } 48 \% \\
\text { Mean age: } 31.9 \\
\text { years }\end{array}$ & MBI-HSS & $\begin{array}{l}\text { Non } \\
\text { medical: } \\
16.5(\mathrm{NR}) \\
\text { Medical: } \\
26.9(\mathrm{NR})\end{array}$ & $\begin{array}{l}7.43 \\
(\mathrm{NR}) \\
10.9 \\
(\mathrm{NR})\end{array}$ & $\begin{array}{l}28.5 \\
(\mathrm{NR}) \\
39.9(\mathrm{NR})\end{array}$ & $\begin{array}{l}\text { Presence of } \\
\text { moderate to } \\
\text { high burnout: } \\
28 \% \text { in non } \\
\text { medical MHPs } \\
52 \% \text { in early } \\
\text { career } \\
\text { psychiatrists }\end{array}$ & 5 \\
\hline
\end{tabular}

NS not significant, NR Not reported, ${ }^{*} \mathrm{p}<0.05,{ }^{* *} \mathrm{p}<0.01,{ }^{* * *} \mathrm{p}<0.001$.

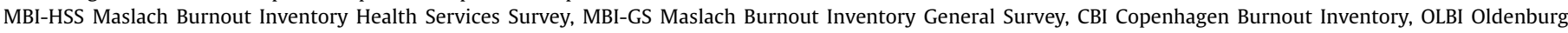
Burnout Inventory, ProQOL Professional Quality of Life. 
the Professional Quality of Life Scale (ProQOL III) [85] $(\mathrm{n}=1)$ and the Children's Services Survey- emotional exhaustion subscale $(n=1)$. Five studies utilised more than one validated measure of burnout.

The MBI-Human Services Survey (MBI-HSS) was utilised by 50 studies while the MBI-General Survey (MBI-GS) was utilised by four studies (See Table 2). The original MBI-HSS was developed for the human services field and included 22 items; emotional exhaustion (MBI-EE nine items), depersonalisation (MBI-DP five items), personal accomplishment (MBI-PA eight items). The scores for each of the three factors are totalled separately and can be coded as low, average or high using cut-off scores defined in the MBI Manual [83]. See Appendix 3 for information on the cut-off scores for MHPs. Reliability and validity of the MBI-HSS have been established across a wide range of countries and professional settings including in the mental health field [83,86-89]. Maslach and Jackson later adopted a measure suitable for use in any professional context the MBI-General Survey (MBI- GS). This MBIGS contains three scales that parallel those of the original MBI: Exhaustion (EX), Cynicism (CY) and Personal Efficacy (PE). This scale has been found to be reliable and valid across multiple occupational and cultural settings [90].

\subsection{Prevalence of burnout in MHPs}

\subsubsection{Mean score on MBI subscales}

Thirty-nine studies reported means and standard deviations for the different dimensions of burnout while five studies reported means but no standard deviations. Only studies, which utilised the MBI-HSS, and the MBI-GS were included in the meta-analysis (33 studies). The total sample of MHPs was $n=9409$. The overal random-effects estimate of the mean for the MBI-EE was 21.25 (95\% CI 19.92, 22.58, MBI-DP was 6.82 (95\% CI 6.13, 7.48) and MBIPA was 34.61 (95\% CI 32.97, 41, 24). There was significant evidence of between-study heterogeneity (EE: Q 1282.8, df 36, $\mathrm{p}<0.001$; $\mathrm{I}^{2}=97.3 \%$, DP: Q 1485.0, df 33, p < 0.001; $\mathrm{I}^{2}=97.8 \%$, PA: Q 5577, df $34, \mathrm{p}<0.001, \mathrm{I}^{2}=99.39 \%$ ). See Fig. 2 for forest plots. Sensitivity analyses, in which the meta-analysis was serially repeated after exclusion of each study, demonstrated that no individual study affected the overall pooled mean by more than 0.50 point (See Appendix 4). To further characterise the range of MBI subscale mean estimates, some pre-defined subgroup analyses and metaregression analyses were conducted.

When only studies rated 'good' on NOS (M8) were considered, the pooled mean estimates decreased for EE to 17.54 (95\%CI 16.27 , $18.02)$, with reduced heterogeneity $\left(\mathrm{I}^{2}=73 \%, \mathrm{p}<0.001\right)$, for DP to 5.19 (95\%CI 5.05, 5.34) with reduced heterogeneity $\left(\mathrm{I}^{2}=83 \%\right.$, $\mathrm{p}<0.001$ ) and for PA to 37.81 (95\% CI 37.37, 37.96, $\mathrm{I}^{2}=96.3 \%$, $\mathrm{P}<0.001$ ) (Appendix 5). When the studies were analysed in subgroups according to the geographical region in which they were conducted there were significant differences noted across the PA mean estimates (test for subgroup differences Q 59.17, $\mathrm{p}<0.001$ ). When only studies from North America were considered, the pooled mean estimates for PA increased to 41.74 (95\% CI 41.52, 41.93) ( $\left.\mathrm{I}^{2}=99 \%, \mathrm{p}<0.001\right)$, whereas when only studies from Europe were considered, the pooled mean estimate for PA reduced to 32.49 (95\% CI 32.29, 32.69) $\left(\mathrm{I}^{2}=99 \%, \mathrm{p}<0.001\right)$ (Appendix 5).

Meta regression analyses indicated that age was associated with increased PA, (slope $=0.36$ points increase on the PA scale per 1 -year increase in average age [95\% CI 0.11 to 0.62 ]; $Q=6.52$, $p=0.01 ; R^{2}=0.52$ ). Estimates of the pooled mean of EE was found to vary with study size (slope $=-0.01$ point reduction in the EE mean, per increase of $n=1$ [95\% CI, -0.01 to -0.0004$] ; Q=4.53$, $\left.\mathrm{p}=0.03 ; \mathrm{R}^{2}=0.03\right]$. Estimates of the pooled mean EE and DP were found to vary with the percentage of nurses in the study (slope= -0.02 point decrease in EE mean, per $1 \%$ increase in nurses in the sample [ $95 \% \mathrm{CI}-0.04$ to 0.002 ]; $\mathrm{Q}=4.8, \mathrm{p}=0.02, \mathrm{R}^{2}=0.17$ ), ( lope $=$ -0.01 decrease in DP mean per $1 \%$ increase in nurses in the sample [95\% CI -0.02 to -0.003 ]; $\left.Q=7.01, \quad \mathrm{p}=0.008, \mathrm{R}^{2}=0.27\right]$. The percentage of psychologists in a study was also found to be associated with decreased DP and increased PA scores (slope = -0.004 decrease in DP score with each increase in $1 \%$ of psychologists in the sample [95\% CI -0.08 to 0.00 ]; $Q=3.84$, $\mathrm{p}<0.001, \mathrm{R}^{2}=0.66$ ), (slope $=0.01$ increase in PA score with each increase in $1 \%$ of psychologists in the sample [95\% CI 0.011 to 0.013 ] $\mathrm{Q}=622.8, \mathrm{R}^{2}=1$ ). See Appendix 6 .

\subsubsection{Prevalence of 'high' rates on burnout subscales}

The meta-analytic pooling of the prevalence estimates of 'high' rates of emotional exhaustion, 'high' rates of depersonalisation and 'low' rates of personal accomplishment were calculated for studies utilising the MBI-HSS (15 studies) and MBI-GS (2 studies). Where the 'cut off' was unclear or was not in line with those recommended by the MBI scale authors, this was stated in Table 1 and the study was not included in the meta-analysis. Seventeen studies reported on 'high' rates for emotional exhaustion $(n=7935)$ and fourteen studies reported on 'high' rates for depersonalisation/ cynicism and personal accomplishment / personal efficacy $(\mathrm{n}=7469)$. The pooled prevalence indicated that $40 \%\left(\mathrm{CI} 31 \%-48 \%, \mathrm{Q}=4874, \mathrm{df}=13, \mathrm{p}<0.001, \mathrm{I}^{2}=99.7\right)$ exceeded the 'high' cut-off for emotional exhaustion, $22 \%$ (CI $15 \%-29 \%$, $\left.\mathrm{Q}=64710, \mathrm{p}<0.001, \mathrm{I}^{2}=99.9\right)$ exceeded 'high' cut-off for depersonalisation / cynicism and 19\% (CI 13\%-25\%, Q = 2605, $\mathrm{p}<0.001$, $\mathrm{I}^{2}=99.7$ ) exceeded cut-off for low levels of personal accomplishment/ personal efficacy. See Fig. 3. There was significant evidence of between-study heterogeneity, and subgroup analyses and metaregression analyses were conducted to explore this.

Studies included in this meta-analysis applied two different 'cutoff points on the MBI-HSS when determining prevalence rates. Eleven studies applied the cut-off specified for MHPs $(\mathrm{EE}>21, \mathrm{DP}>8$, $\mathrm{PA}<28)[27,29,31,32,39,53,58,63,70,74]$, three studies utilised the cut-offs for other health professionals (EE 27, EDP 11, PA 835 ) $[48,49,78]$. A subgroup analysis was conducted to investigate the extent the use of two different cut-offs points was contributing to the between-study heterogeneity. The pooled prevalence of the EE $>21$ cut-off group $(n=2542)$ was estimated at $44 \%(95 \% \mathrm{CI}=38 \%-49 \%)$ and for the EEc26 cut-off group ( $n=945)$ was estimated at 21\% (95\% $\mathrm{CI}=8 \%-33 \%$ ). This was a statistically significant difference between the two groups $(\mathrm{Z} 13.46, \mathrm{p}<0.001)$. The pooled prevalence of the $D P>8$ group $(n=1735)$ was estimated at $26 \%(95 \% C I=20 \%-33 \%)$ and the pooled prevalence of the DPE11 $(n=945)$ group was $9 \%(95 \%$ $\mathrm{CI}=5 \%-12 \%)$, a statistically significant difference $(\mathrm{Z} 10.29, \mathrm{p}<0.001)$. The pooled prevalence of the $P A<28$ group $(n=1519)$ was estimated to be $18 \%(95 \% \mathrm{CI}=9 \%-28 \%)$ and for the PA $\delta 35$ group $(\mathrm{n}=945)$ was estimated to be $27 \%$ ( $95 \% \mathrm{CI}=21 \%-33 \%$. This difference was also statistically significant $(Z 6.26, p<0.001)$. See Appendix 7. A metaregression analysis found that more than $50 \%$ of the EE betweenstudy heterogeneity and more than 40\% DP and PA between-study heterogeneity may be explained by the use of the two different MBIHSS cut off scores (EE coefficient $=25.04,95 \% \mathrm{CI}=14.8-35.3$, $\mathrm{p}<0.001, \quad \mathrm{R}^{2=0.52 ;}$ DP coefficient $=16.86,95 \%$ CI 5.66-28.06, $\mathrm{P}<0.001, \mathrm{R}^{2}=0.44 ;$ PA coefficient $=26.23,95 \%$ ci $20.37,32.08$, $\left.\mathrm{p}<0.001, \mathrm{R}^{2}=0.44\right)$

\subsection{Publication bias}

Inspection of the funnel plots demonstrated that studies were distributed symmetrically. The Eggers test was not significant for bias for the means/ prevalence of emotional exhaustion $(t=1.43$, $\mathrm{df}=31, \mathrm{p}=0.08)$ depersonalisation $(\mathrm{t}=1.94, \mathrm{df}=33 \mathrm{p}=0.06)$ or professional accomplishment $(\mathrm{t}=1.37, \mathrm{df}=31, \mathrm{p}=0.10$ ) (See Appendix 8). 
Table 2

Determinants of Burnout in Mental Health Professionals.

\begin{tabular}{|c|c|c|c|c|c|c|c|}
\hline Study & Design & $\begin{array}{l}\text { No of } \\
\text { Resp }\end{array}$ & $\begin{array}{l}\text { Measure of } \\
\text { Burnout }\end{array}$ & Measure of determinants & $\mathrm{EE}$ & DP & $\mathrm{PA}$ \\
\hline [21] & CS & $N=140$ & MBI- HSS & Gender (female) & $\beta=3.2^{*}$ & NS & NS \\
\hline Angermeyer & & & & Married & NS & NS & $\beta-3.65^{*}$ \\
\hline et al. (2006) & & & & Intensity of care & $\beta=0.059^{*}$ & $\beta=0.027^{*}$ & NS \\
\hline $\begin{array}{l}\text { [22] Ashtari } \\
\text { et al. (2009) }\end{array}$ & CS & $\mathrm{N}=100$ & MBI- HSS & Job Performance Inventory & $r=0.60^{* *}$ & $\mathrm{r}=0.57^{* *}$ & $r=0.66^{* *}$ \\
\hline [23] Benbow & CS & $\mathrm{N}=145$ & MBI-HSS & Age & NS & $\mathrm{r}=-0.35^{* * *}$ & NS \\
\hline $\begin{array}{l}\text { \& Jolley } \\
(2002)\end{array}$ & & & & Stress checklist & $\mathrm{r}=0.701^{* * *}$ & $\mathrm{r}=0.544^{* * *}$ & $r=-0.487$ \\
\hline [24] & $\mathrm{CS}$ & $N=363$ & MBI & Gender (female) & NS & $\mathrm{r}=-1.3^{* *}$ & NS \\
\hline \multirow{8}{*}{$\begin{array}{l}\text { Billings et al. } \\
(2003)\end{array}$} & & & & Black ethic group & $\mathrm{r}=-6.4^{* *}$ & $\mathrm{r}=-2.0^{*}$ & NS \\
\hline & & & & Age over 55years & NS & $\mathrm{r}=-8.3^{*}$ & NS \\
\hline & & & & Psychiatrist & $r=-5.0^{*}$ & NS & NS \\
\hline & & & & Psychologist & NS & $\mathrm{r}=-3.2^{* *}$ & NS \\
\hline & & & & Time in current post & $r=-0.62^{*}$ & $r=0.24^{*}$ & NS \\
\hline & & & & Time as mental health worker & NS & $\mathrm{r}=-0.11^{*}$ & NS \\
\hline & & & & Assertive outreach team member & NS & $\mathrm{r}=-1.7^{*}$ & $\mathrm{r}=1.8^{*}$ \\
\hline & & & & $\begin{array}{l}\text { Minnesota job satisfaction scale \& job } \\
\text { diagnostic survey (job satisfaction) }\end{array}$ & NR & NR & NR \\
\hline \multirow{5}{*}{$\begin{array}{l}{[25]} \\
\text { Blau et al. } \\
(2013)\end{array}$} & CS & $N=1639$ & MBI-HSS & Age & NS & $\beta=-0.05^{*}$ & $\beta=0.04^{*}$ \\
\hline & & & & Gender (female) & NS & $\beta=0.20^{* *}$ & NS \\
\hline & & & & Education level & $\beta=0.08^{*}$ & NS & NS \\
\hline & & & & Personal involvement & NS & $\beta=0.05^{* *}$ & NS \\
\hline & & & & Length of service & NS & NS & $\beta=-0.05^{*}$ \\
\hline \multirow{22}{*}{$\begin{array}{l}{[26]} \\
\text { Bowers et al } \\
(2009)\end{array}$} & CS & $N=1525$ & MBI & Gender (female) & $\mathrm{R}=-2.19^{* * *}$ & $\mathrm{R}=-0.93^{* *}$ & NS \\
\hline & & & & One year or less in current post & $\mathrm{R}=-2.54^{* *}$ & NS & NS \\
\hline & & & & Between 3 and 5 years in current post & $\mathrm{R}=2.21^{*}$ & NS & NS \\
\hline & & & & 20 or under compared to $30-39$ years of age & NS & $\mathrm{R}=3.85^{*}$ & NS \\
\hline & & & & $40-49$ compared to $30-39$ years of age & NS & $\mathrm{R}=1.62^{* * *}$ & NS \\
\hline & & & & $50-59$ compared to $30-39$ years of age & $\mathrm{R}=-2.23^{*}$ & $\mathrm{R}=-1.62^{* * *}$ & NS \\
\hline & & & & 60 and over compared to $30-39$ years of age & $\mathrm{R}=-5.96^{* *}$ & $\mathrm{R}=-2.05^{*}$ & NS \\
\hline & & & & Working between 1 and 2 years in psychiatry & $\mathrm{R}=1.38^{*}$ & NS & NS \\
\hline & & & & compared to 5 years & $\mathrm{R}=-1.13^{* *}$ & NS & NS \\
\hline & & & & African compared to white & $\mathrm{R}=-2.261^{*}$ & NS & NS \\
\hline & & & & Access to ACT team & NS & $\mathrm{R}=0.023^{*}$ & NS \\
\hline & & & & Index of multiple deprivation (patient) & NS & NS & NS \\
\hline & & & & Patient-staff Conflict Checklist & NS & $\mathrm{R}=-0.325^{* *}$ & NS \\
\hline & & & & Attitudes to Containment Measures & NS & $\mathrm{R}=0.229^{*}$ & NS \\
\hline & & & & Questionnaire & NS & $\mathrm{R}=-1.815^{*}$ & NS \\
\hline & & & & - Safe for patients & $\mathrm{R}=4.375^{* *}$ & $\mathrm{R}=1.508^{*}$ & NS \\
\hline & & & & - Prepared to use & $\mathrm{R}=4.755^{* *}$ & $R=-1.612^{*}$ & $\mathrm{R}=1.806^{*}$ \\
\hline & & & & Attitude to Personality Disorder & $\mathrm{R}=-3.943^{* *}$ & NS & NS \\
\hline & & & & Questionnaire & NS & NS & $\mathrm{R}=1.497^{*}$ \\
\hline & & & & - Acceptance & NS & $\mathrm{R}=-0.361^{* *}$ & $\mathrm{R}=0.615^{* *}$ \\
\hline & & & & $\begin{array}{l}\text { - Purpose } \\
\text { - Enthusiasm } \\
\text { - Security } \\
\text { - Enjoyment } \\
\text { Ward Atmosphere Scale } \\
\text { - Order and organisation }\end{array}$ & NS & NS & NS \\
\hline & & & & $\begin{array}{l}\text { Multifactor Leadership Questionnaire (MLQ- } \\
\text { X4) }\end{array}$ & & & \\
\hline \multirow{5}{*}{$\begin{array}{l}{[27]} \\
\text { Bressi et al. } \\
(2009)\end{array}$} & CS & $\mathrm{N}=81$ & MBI-HSS & GHQ-12 & NS & NS & NS \\
\hline & & & & Job Diagnostic Survey & $\beta=0.17^{*}$ & NS & NS \\
\hline & & & & Work with patients family & $\beta=-0.49^{* * *}$ & $\beta=-0.26^{*}$ & $\beta=0.31^{*}$ \\
\hline & & & & Job satisfaction & NS & $\beta=0.26^{*}$ & NS \\
\hline & & & & $\begin{array}{l}\text { Work with demanding patients } \\
\text { Negative relationship with patients }\end{array}$ & NS & NS & $\beta=-0.23^{*}$ \\
\hline \multirow{7}{*}{$\begin{array}{l}{[28]} \\
\text { Chakraborty } \\
\text { et al. (2012) }\end{array}$} & $\mathrm{CS}$ & $\mathrm{N}=101$ & $\mathrm{CBI}$ & Age & Burnout & - & - \\
\hline & & & & Duration period of nursing & $-0.236^{*}$ & - & - \\
\hline & & & & Duration of army service & $-0.252^{*}$ & - & - \\
\hline & & & & Emotional maturity scale & $-0.332^{*}$ & - & - \\
\hline & & & & General well being scale & $-0.554^{* * *}$ & - & - \\
\hline & & & & Locus of control scale & $-0.403^{* * *}$ & & \\
\hline & & & & & $-0.280^{* *}$ & & \\
\hline \multirow{7}{*}{$\begin{array}{l}{[30]} \\
\text { Devilly et al. } \\
(2009)\end{array}$} & CS & $N=152$ & $\mathrm{CBI}$ & Post-traumatic Stress Scale (adapted) & NR & NR & NR \\
\hline & & & & Depression, Anxiety and Stress Scale & NR & NR & NR \\
\hline & & & & Secondary Traumatic Stress Scale & NR & NR & NR \\
\hline & & & & TSI Belief Scale- revision L & NR & NR & NR \\
\hline & & & & Interpersonal Reactivity Index & NR & NR & NR \\
\hline & & & & Interpersonal Support Evaluation List & NR & NR & NR \\
\hline & & & & & $\begin{array}{l}\text { Exposure to service users } \\
\text { traumatic experiences did not } \\
\text { affect burnout rates. }\end{array}$ & & \\
\hline
\end{tabular}


Table 2 (Continued)

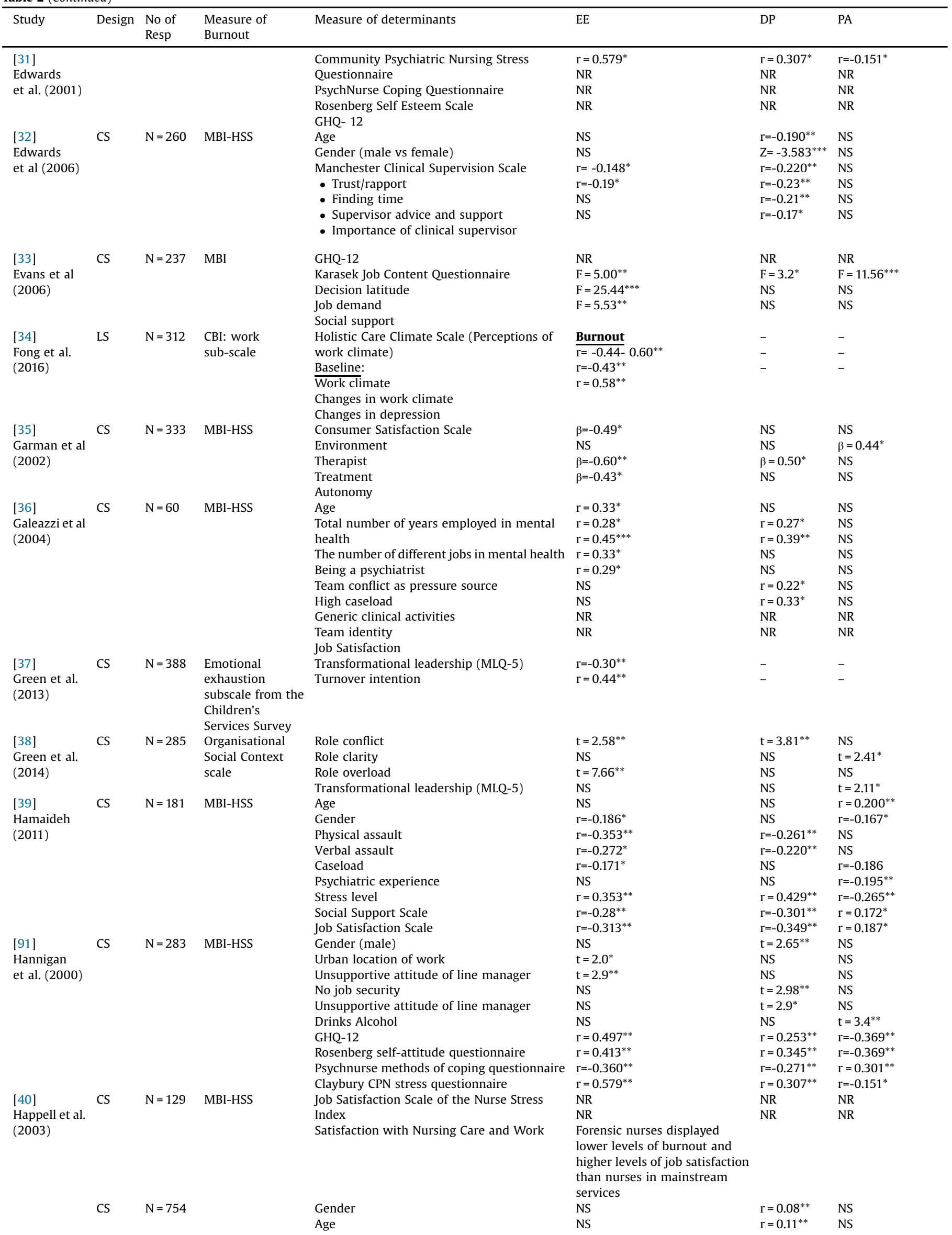


Table 2 (Continued)

\begin{tabular}{|c|c|c|c|c|c|c|c|}
\hline Study & Design & $\begin{array}{l}\text { No of } \\
\text { Resp }\end{array}$ & $\begin{array}{l}\text { Measure of } \\
\text { Burnout }\end{array}$ & Measure of determinants & $\mathrm{EE}$ & DP & PA \\
\hline $\begin{array}{l}\text { [44] } \\
\text { Jeanneau \& } \\
\text { Armelius } \\
\text { [45] } \\
\text { Johnson } \\
\text { et al. (2012) }\end{array}$ & $\mathrm{CS}$ & $N=2258$ & $\begin{array}{l}\text { MBI } \\
\text { Pines Burnout } \\
\text { Measure } \\
\text { MBI-HSS }\end{array}$ & $\begin{array}{l}\text { Job related affective well being scale } \\
\text { Anxiety contentment } \\
\text { Depression-enthusiasm } \\
\text { GHQ-12 } \\
\text { Workplace employment relations survey- } \\
\frac{2004}{\text { NHS staff survey }} \\
\text { Job Involvement Scale } \\
\text { Intrinsic satisfaction } \\
\text { Differences across service type } \\
\text { Difference across profession }\end{array}$ & $\begin{array}{l}\frac{E x}{\mathrm{r}=-0.63^{* * *}} \\
\mathrm{r}=-0.64^{* * *} \\
\mathrm{r}=0.63^{* * *} \\
\mathrm{r}=-0.12^{* * *} \\
\mathrm{r}=-0.45^{* * *} \\
\mathrm{~F}=8.97^{* * *} \\
\mathrm{EE} \text { highest in CMHT }(21.1+/- \\
12.7) \\
\text { lowest in CRHT }(17.7+/-10.7) \\
\mathrm{F}=6.65^{* * *} \\
\mathrm{EE} \text { highest in social workers }(23 \\
+/-12.15) \\
\text { lowest in 'other' occupations } \\
(17.2+/-10.87)\end{array}$ & $\begin{array}{l}\text { Cynicism } \\
r=0.55^{* * * *} \\
r=-0.40^{* * *} \\
r=0.40^{* * *} \\
r=-0.12^{* * *} \\
r=-0.12^{* * *} \\
N R \\
N R\end{array}$ & $\begin{array}{l}\text { Personal } \\
\text { Accomplishment } \\
\mathrm{r}=0.27^{* * *} \\
\mathrm{r}=0.39^{* * *} \\
\mathrm{r}=-0.27^{* * *} \\
\mathrm{r}=0.17^{* * *} \\
\mathrm{r}=0.17^{* * *} \\
\mathrm{~F}=4.38^{* *} \\
\text { PA highest in } \\
\text { rehabilitation } \\
\text { wards (35.1 } \\
+/-7.9) \\
\text { lowest in forensic } \\
\text { wards (32.1 +/- } \\
8.9 \text { ) } \\
\mathrm{F}=2.87^{* *} \\
\text { PA highest in } \\
\text { service managers } \\
(35.8+/-6.38) \\
\text { lowest in nursing } \\
\text { assistants (32.7 } \\
+/-9.27 \text { ) }\end{array}$ \\
\hline $\begin{array}{l}\text { [46] } \\
\text { Johnson } \\
\text { et al. (2016) }\end{array}$ & CS & $\mathrm{N}=114$ & MBI- HSS & $\begin{array}{l}\text { Measure of trust within teams } \\
\text { - Propensity to trust } \\
\text { - Perceived trust } \\
\text { - Cooperative behaviours } \\
\text { - Monitoring behaviours } \\
\text { Boundary violations- scale developed by } \\
\text { authors } \\
\text { - Frequency of boundary violations } \\
\text { - Impact of boundary violation }\end{array}$ & $\begin{array}{l}\text { NS } \\
-0.305^{* *} \\
-0.338^{* *} \\
0.213^{*} \\
\text { NS } \\
0.233^{*}\end{array}$ & $\begin{array}{l}\text { NS } \\
0.071 \\
-0.271^{* *} \\
0.291^{*} \\
0.191^{*} \\
0.201^{*}\end{array}$ & $\begin{array}{l}-0.277^{* *} \\
-0.275^{* *} \\
\text { NS } \\
\text { NS } \\
\text { NS } \\
\text { NS }\end{array}$ \\
\hline $\begin{array}{l}\text { [16] } \\
\text { Jovanovic } \\
\text { et al. (2016) }\end{array}$ & $\mathrm{CS}$ & $N=1980$ & MBI-GS & $\begin{array}{l}\text { Age } \\
\text { Gender (males) } \\
\text { Psychiatry not first career choice } \\
\text { No postgraduate education } \\
\text { Working hours } \\
\text { Not enough daily rest } \\
\text { No clinical supervision }\end{array}$ & $\begin{array}{l}\frac{\mathbf{E x}}{\mathrm{NS}} \\
\mathrm{NS} \\
\mathrm{NS} \\
\mathrm{NS} \\
\mathrm{r}=0.11^{* * *} \\
\mathrm{r}=0.33^{* * *} \\
\mathrm{r}=0.34^{* * *}\end{array}$ & $\begin{array}{l}\text { Cynicism } \\
r=-0.13^{* * *} \\
r=0.14^{*} \\
r=0.22^{* *} \\
\text { NS } \\
\text { NS } \\
\text { NS } \\
r=0.34^{* * *}\end{array}$ & $\begin{array}{l}\text { Personal } \\
\text { Accomplishment } \\
\text { NS } \\
\text { NS } \\
\text { NS } \\
\text { r=-0.15** } \\
\text { NS } \\
\text { NS } \\
\text { NS }\end{array}$ \\
\hline $\begin{array}{l}{[47]} \\
\text { Karanikola } \\
\text { et al. (2013) }\end{array}$ & $\mathrm{CS}$ & $N=226$ & MBI-HSS & $\begin{array}{l}\text { Hamilton Anxiety Scale } \\
\text { Beck Depression Scale }\end{array}$ & $\begin{array}{l}r=0.562^{* * *} \\
r=0.616^{* * *}\end{array}$ & $\begin{array}{l}\mathrm{r}=0.448^{* * *} \\
\mathrm{r}=0.394^{* * *}\end{array}$ & $\begin{array}{l}\text { NS } \\
r=-0.186^{* *}\end{array}$ \\
\hline $\begin{array}{l}{[48]} \\
\text { Kilfedder } \\
\text { et al (2001) }\end{array}$ & CS & $\mathrm{N}=635$ & MBI & $\begin{array}{l}\text { Length in post } \\
\text { GHQ-12 } \\
\text { Job satisfaction survey } \\
\text { Psychosomatic symptoms } \\
\text { Positive and Negative Affect Schedule } \\
\text { Positive affectivity } \\
\text { Negative affectivity } \\
\text { Social support measure } \\
\text { Predictability } \\
\text { Job future ambiguity measure } \\
\text { Role ambiguity } \\
\text { measure } \\
\text { Role conflict measure } \\
\text { Nursing stress scale }\end{array}$ & $\begin{array}{l}\text { NS } \\
\beta=0.5^{* *} \\
\beta=-0.2^{* *} \\
\beta=0.7^{* *} \\
\beta=0.3^{* *} \\
\beta=-0.5^{*} \\
\beta=-0.3^{* *} \\
\beta=-0.3^{* *} \\
\beta=-0.3^{* *} \\
\beta=-0.3^{* *} \\
\beta=0.3^{* *} \\
\beta=0.4^{* *}\end{array}$ & $\begin{array}{l}\text { NS } \\
\beta=0.2^{* *} \\
\text { NS } \\
\beta=0.3^{* *} \\
\beta=0.2^{* *} \\
\beta=0.3^{* *} \\
\beta=-0.2^{* *} \\
\beta=-0.2^{* *} \\
\beta=-0.2^{* *} \\
\beta=-0.2^{* *} \\
\beta=0.2^{* *} \\
\beta=0.3^{* *}\end{array}$ & $\begin{array}{l}\beta=-0.2^{* *} \\
\text { NS } \\
\text { NS } \\
\text { NS } \\
\beta=0.3^{* *} \\
\text { NS } \\
\beta=0.2^{* *} \\
\beta=0.2^{* *} \\
\beta=0.2^{* *} \\
\beta=0.2^{* *} \\
\text { NS } \\
\text { NS }\end{array}$ \\
\hline $\begin{array}{l}{[49]} \\
\text { Kumar et al. } \\
(2007)\end{array}$ & CS & 239 & MBI-HSS & $\begin{array}{l}\text { Gender } \\
\text { Marital status } \\
\text { Years of practice } \\
\text { Job diagnostic survey }\end{array}$ & $\begin{array}{l}\text { NS } \\
\mathrm{F}=5.75^{* * *} \\
\mathrm{NS} \\
\mathrm{p}=-0.38^{* *}\end{array}$ & $\begin{array}{l}\text { NS } \\
\text { NS } \\
F=3.5^{*} \\
p=-0.38^{* *}\end{array}$ & $\begin{array}{l}\mathrm{F}=4.22^{*} \\
\mathrm{NS} \\
\mathrm{NS} \\
\mathrm{p}=0.29^{* *}\end{array}$ \\
\hline $\begin{array}{l}{[50]} \\
\text { Kumar et al. } \\
(2011)\end{array}$ & LS & $N=131$ & $\begin{array}{l}\text { MBI- HSS EE score } \\
\text { only }\end{array}$ & $\begin{array}{l}\text { Stress (Sources of stress questionnaire- } \\
\text { developed by authors) } \\
\text { - Too much work } \\
\text { - Too long working hours } \\
\text { - Aggressive administrative environment } \\
\text { - Lack of support from management }\end{array}$ & $\begin{array}{l}\mathrm{p}<0.05 \\
\mathrm{p}<0.05 \\
\mathrm{p}<0.05 \\
\mathrm{p}<0.05\end{array}$ & $\begin{array}{l}- \\
- \\
-\end{array}$ & $\begin{array}{l}- \\
- \\
-\end{array}$ \\
\hline $\begin{array}{l}{[52]} \\
\text { Lloyd and } \\
\text { King }\end{array}$ & $\mathrm{CS}$ & $N=304$ & MBI-HSS & $\begin{array}{l}\text { Younger Age } \\
\text { Greater amount of activity in general clinical } \\
\text { work }\end{array}$ & $\begin{array}{l}\text { NS } \\
\text { NS }\end{array}$ & $\begin{array}{l}\mathrm{F}=7.8^{* *} \\
\mathrm{NS}\end{array}$ & $\begin{array}{l}\mathrm{NS} \\
\mathrm{F}=16.0^{* * *}\end{array}$ \\
\hline
\end{tabular}


Table 2 (Continued)

\begin{tabular}{|c|c|c|c|c|c|c|c|}
\hline Study & Design & $\begin{array}{l}\text { No of } \\
\text { Resp }\end{array}$ & $\begin{array}{l}\text { Measure of } \\
\text { Burnout }\end{array}$ & Measure of determinants & $\mathrm{EE}$ & DP & PA \\
\hline $\begin{array}{l}{[51]} \\
\text { Lasalvia et al } \\
(2009)\end{array}$ & $\mathrm{CS}$ & $N=1585$ & MBI-GS & $\begin{array}{l}\text { Areas of Worklife Scale (AWS) } \\
\text { - Workload } \\
\text { - Control } \\
\text { - Reward } \\
\text { - Fairness } \\
\text { Evaluation of Changes scale } \\
\text { Management Areas scale } \\
\text { - Skills development } \\
\text { - Work-group cohesion }\end{array}$ & $\begin{array}{l}\frac{\mathbf{E x}}{\mathbf{R}(\mathbf{S E})} \\
1.08(0.07)^{* *} \\
-0.15(0.07)^{*} \\
-0.22(0.06)^{* *} \\
\text { NS } \\
0.35(0.09)^{* *} \\
-0.18(0.07)^{* *} \\
\text { NS }\end{array}$ & $\begin{array}{l}\text { Cyn } \\
\mathbf{R}(\mathbf{S E}) \\
0.28(0.07) \\
* * \\
-0.25 \\
(0.07)^{* *} \\
-0.35 \\
(0.06)^{* *} \\
-0.29 \\
(0.09)^{* *} \\
-0.39 \\
(0.09)^{* *} \\
-0.22 \\
(0.07)^{* *} \\
0.19(0.07)^{* *}\end{array}$ & $\begin{array}{l}\text { Efficacy } \\
\text { R(SE) } \\
\text { NS } \\
0.55(0.06)^{* *} \\
0.24(0.06)^{* *} \\
0.28(0.08)^{* *} \\
\text { NS } \\
\text { NS } \\
0.20(0.07)^{* *}\end{array}$ \\
\hline $\begin{array}{l}{[53]} \\
\text { Levert et al } \\
(2001)\end{array}$ & $\mathrm{CS}$ & $\mathrm{N}=94$ & MBI-HSS & $\begin{array}{l}\text { Work load (Workload and lack of collegial } \\
\text { support) } \\
\text { - Work load } \\
\text { - Collegial support } \\
\text { Role conflict \& ambiguity ( } \\
\text { - Role conflict } \\
\text { - Role ambiguity } \\
\text { Antonovsky's sense of coherence (Orientation } \\
\text { to life questionnaire) }\end{array}$ & $\begin{array}{l}r=0.47^{* * *} \\
r=0.21^{*} \\
r=0.36^{* * *} \\
r=0.29^{* *} \\
r=0.41^{* * *}\end{array}$ & $\begin{array}{l}r=0.31^{* * *} \\
r=0.23^{*} \\
r=0.3^{* * *} \\
r=0.31^{* * *} \\
r=0.36^{* *}\end{array}$ & $\begin{array}{l}\text { NS } \\
\text { NS } \\
\text { NS } \\
r=0.26^{*} \\
\text { NS }\end{array}$ \\
\hline $\begin{array}{l}\text { [54] } \\
\text { Madathil } \\
\text { et al. (2014) } \\
\text { USA }\end{array}$ & CS & $N=89$ & MBI-HSS & $\begin{array}{l}\text { MLQ- X4 } \\
\text { Nursing Work Index-revised } \\
\text { Brief symptom inventory }\end{array}$ & $\begin{array}{l}\text { NR } \\
\text { NR } \\
\text { NR } \\
\text { Leadership style and work role } \\
\text { autonomy protect nurses } \\
\text { againsy burnout. }\end{array}$ & $\begin{array}{l}\text { NR } \\
\text { NR } \\
\text { NR }\end{array}$ & $\begin{array}{l}\text { NR } \\
\text { NR } \\
\text { NR }\end{array}$ \\
\hline $\begin{array}{l}{[55]} \\
\text { Melchior } \\
\text { et al. (1997) }\end{array}$ & CS & $\mathrm{N}=361$ & MBI- HSS & $\begin{array}{l}\text { Age } \\
\text { Work experience in nursing } \\
\text { Job characteristics } \\
\text { (Adapted job diagnostic survey) } \\
\text { complexity } \\
\text { autonomy } \\
\text { feedback/clarity } \\
\text { Leadership behaviour questionnaire } \\
\text { social } \\
\text { instrumental } \\
\text { Nursing care model questionnaire } \\
\text { personal care tasks } \\
\text { psycho-social tasks } \\
\text { household tasks } \\
\text { organizational tasks }\end{array}$ & $\begin{array}{l}\text { NS } \\
\text { NS } \\
r=0.28^{* * *} \\
\text { NS } \\
r=-0.18^{* * *} \\
r=0.22^{* * *} \\
\text { NS } \\
\text { NS } \\
\text { NS } \\
r=0.11^{*} \\
r=0.15^{* *}\end{array}$ & $\begin{array}{l}\text { NS } \\
\text { NS } \\
r=0.23^{* * *} \\
\text { NS } \\
r=-0.19^{* * *} \\
r=-0.19^{* * *} \\
\text { NS } \\
\text { NS } \\
r=0.13^{*} \\
r=0.15^{* *} \\
\text { NS }\end{array}$ & $\begin{array}{l}r=0.15^{* *} \\
r=0.15^{* *} \\
N S \\
r=0.18^{* * *} \\
r=0.34^{* * *} \\
r=0.16^{* *} \\
\text { NS } \\
\text { NS } \\
\text { NS } \\
\text { NS } \\
\text { NS }\end{array}$ \\
\hline $\begin{array}{l}{[56]} \\
\text { Ndetei et al. } \\
\text { (2008) }\end{array}$ & CS & $N=285$ & MBI- HSS & $\begin{array}{l}\text { Work attitudes and relationships } \\
\text { (questionnaire developed by study authors) }\end{array}$ & $\begin{array}{l}\text { NR } \\
\text { Young age, number of own } \\
\text { children, number of years } \\
\text { worked, workload and low } \\
\text { morale associated with } \\
\text { burnout. }\end{array}$ & NR & NR \\
\hline $\begin{array}{l}{[57]} \\
\text { Nelson et al } \\
\text { (2009) }\end{array}$ & CS & $\mathrm{N}=132$ & MBI- HSS & $\begin{array}{l}\text { Minnesota Satisfaction Scale } \\
\text { Job Diagnostic Survey- general job subscale } \\
\text { Pan London Assertive Outreach Study } \\
\text { questionnaire on stress and satisfaction } \\
\text { Asian ethnic group (less EE, DP, greater PA) } \\
\text { Time as mental health worker (less EE, less DP } \\
\text { with longer career) } \\
\text { CMHT member (greater DP, less PA) } \\
\text { Female (less DP) } \\
\text { Psychologist (less DP) } \\
\text { Age 46-54 (less DP) } \\
\text { Age over } 55 \text { (less DP) } \\
\text { Black Ethnic group (less DP) } \\
\text { Office hours, incl. evenings and weekends, no } \\
\text { overnight (greater DP) } \\
\text { Shifts, telephone on call only (greater DP) } \\
\text { Shifts, plus on call overnight (greater DP) } \\
\text { Time in type of team (greater PA) }\end{array}$ & $\begin{array}{l}\text { NR } \\
\text { NR } \\
\text { Regression coefficient }(\mathbf{9 5 \%} \\
\text { CI) } \\
-5.5(-9.0 \text { to }-2.0)^{*} \\
-0.2(-0.4 \text { to }-0.01)^{*} \\
\text { NS } \\
\text { NS } \\
\text { NS } \\
\text { NS } \\
\text { NS } \\
\text { NS } \\
\text { NS } \\
\text { NS } \\
\text { NS } \\
\text { NS }\end{array}$ & $\begin{array}{l}\text { NR } \\
\text { NR } \\
\text { Regression } \\
\text { coefficient } \\
\text { (95\% CI) } \\
-1.9(-3.4 \text { to } \\
-0.4)^{*} \\
-0.1(-0.2 \text { to } \\
-0.01) \\
1.8(0.6- \\
3.0)^{* *} \\
-1.3(-2.2 \text { to } \\
-0.4)^{* *} \\
-2.6(-5.0 \\
\text { to }-0.2)^{*} \\
-3.3(-6.3 \\
\text { to }-0.4)^{*} \\
-4.7(-8.3 \\
\text { to }-1.2)^{* *} \\
-2.1(-3.3 \text { to } \\
-0.9)^{* *} \\
1.6(0.5- \\
2.8)^{* *} \\
1.9(0.4-\end{array}$ & $\begin{array}{l}\text { NR } \\
\text { NR } \\
\text { Regression } \\
\text { coefficient }(95 \% \\
\text { CI) } \\
3.4(0.8 \text { to } 6.0)^{*} \\
\text { NS } \\
-2.1(-4.1 \text { to }-0.1)^{*} \\
\text { NS } \\
\text { NS } \\
\text { NS } \\
\text { NS } \\
\text { NS } \\
\text { NS } \\
\text { NS } \\
\text { NS } \\
0.5(0.2-0.9)^{* *}\end{array}$ \\
\hline
\end{tabular}


Table 2 (Continued)

\begin{tabular}{|c|c|c|c|c|c|c|c|}
\hline Study & Design & $\begin{array}{l}\text { No of } \\
\text { Resp }\end{array}$ & $\begin{array}{l}\text { Measure of } \\
\text { Burnout }\end{array}$ & Measure of determinants & $\mathrm{EE}$ & DP & PA \\
\hline $\begin{array}{l}{[58]} \\
\text { Oddie \& } \\
\text { Ousley } \\
(2007)\end{array}$ & $\mathrm{CS}$ & $N=71$ & MBI-HSS & $\begin{array}{l}\text { Psychiatric Nurse Occupational Stress Scale } \\
\text { - Occupational Stress } \\
\text { - Limited resources } \\
\text { - Staff conflict } \\
\text { - Patient care }\end{array}$ & $\begin{array}{l}\mathrm{r}=0.439^{* *} \\
\mathrm{r}=0.313^{* *} \\
\mathrm{r}=0.287^{*} \\
\mathrm{r}=0.248^{*}\end{array}$ & $\begin{array}{l}3.4)^{*} \\
1.9(0.3- \\
3.5)^{*} \\
N S \\
r=0.419^{* *} \\
r=0.325^{* *} \\
r=0.291^{*} \\
N S\end{array}$ & $\begin{array}{l}\mathrm{r}=-0.383^{* *} \\
\mathrm{NS} \\
\mathrm{r}=-0.337^{*} \\
\text { NS }\end{array}$ \\
\hline $\begin{array}{l}\text { [59] } \\
\text { Ogresta } \\
\text { (2008) } \\
\text { Croatia }\end{array}$ & CS & $\mathrm{N}=174$ & MBI- HSS & $\begin{array}{l}\text { Job Satisfaction Survey } \\
\text { - Rewards } \\
\text { - Work climate } \\
\text { - Advancement and benefits } \\
\text { - Superiors and colleagues } \\
\text { Manifestations of occupational stress ques- } \\
\text { tionnaire } \\
\text { - Psychological manifestations } \\
\text { - Physical manifestations } \\
\text { - Negative emotional and behavioural } \\
\text { reactions towards patients and colleagues }\end{array}$ & $\begin{array}{l}r=-0.52^{* *} \\
r=-0.38^{* *} \\
\text { NS } \\
\text { NS } \\
r=0.45^{*} \\
r=0.40^{*} \\
\text { NS }\end{array}$ & $\begin{array}{l}\text { NS } \\
r=-0.27^{* *} \\
\text { NS } \\
\text { NS } \\
\text { NS } \\
r=0.26^{*} \\
r=0.40^{*}\end{array}$ & $\begin{array}{l}\text { NS } \\
\mathrm{r}=-0.19^{*} \\
\text { NS } \\
\text { NS } \\
\text { NS } \\
\text { NS } \\
\text { NS }\end{array}$ \\
\hline $\begin{array}{l}{[60]} \\
\text { Onyett et al. } \\
(1997)\end{array}$ & CS & $\mathrm{N}=445$ & MBI & $\begin{array}{l}\text { Consultant psychiatrists, social workers, } \\
\text { nurses and psychologists (higher EE) } \\
\text { Consultant psychiatrists (greater DP) } \\
\text { Job Satisfaction Scale } \\
\text { Role ambiguity scale }\end{array}$ & $\begin{array}{l}\mathrm{F}(11,420)=2.9^{* *} \\
\text { NS } \\
\text { NR } \\
\text { NR }\end{array}$ & $\begin{array}{l}\text { NR } \\
\text { K-W } \\
X^{2}=42.5^{* * *} \\
\text { NR } \\
\text { NR }\end{array}$ & $\begin{array}{l}\text { NR } \\
\text { NS } \\
\text { NR } \\
\text { NR }\end{array}$ \\
\hline $\begin{array}{l}{[61]} \\
\text { Oyefeso et al } \\
(2008)\end{array}$ & CS & $N=194$ & MBI-HSS & $\begin{array}{l}\text { GHQ-12 } \\
\text { High levels of alienation } \\
\text { High levels of tension } \\
\text { Age: under } 25\end{array}$ & $\begin{array}{l}\mathrm{NR} \\
\mathrm{OR}=3.49^{* *} \\
\mathrm{OR}=2.65^{*} \\
\mathrm{OR}=7.15^{*}\end{array}$ & $\begin{array}{l}\text { NR } \\
\text { NS } \\
\text { OR }=4.57^{*} \\
\text { NS }\end{array}$ & $\begin{array}{l}\text { NR } \\
\text { NS } \\
\text { NS } \\
\text { NS }\end{array}$ \\
\hline $\begin{array}{l}\text { [62] } \\
\text { Piko (2006 }\end{array}$ & CS & $\mathrm{N}=250$ & MBI & $\begin{array}{l}\text { Gender (male) } \\
\text { Age } \\
\text { Schooling } \\
\text { Psychosomatic symptom scale } \\
\text { Job satisfaction, Role conflict (measure } \\
\text { developed for study) } \\
\text { Job satisfaction } \\
\text { Role conflict } \\
\text { Years in healthcare }\end{array}$ & $\begin{array}{l}\text { NS } \\
\text { NS } \\
\text { NS } \\
R=0.46^{* * *} \\
R=-0.49^{* * *} \\
R=0.35^{* * *} \\
\text { NS }\end{array}$ & $\begin{array}{l}\mathrm{R}=-0.17^{* *} \\
\mathrm{R}=0.19^{*} \\
\mathrm{R}=-0.16^{*} \\
\mathrm{NS} \\
\mathrm{R}=-0.37^{* * *} \\
\mathrm{R}=0.34^{* *} \\
\mathrm{NS}\end{array}$ & $\begin{array}{l}\mathrm{R}=0.26^{* * *} \\
\mathrm{NS} \\
\mathrm{R}=0.14^{*} \\
\mathrm{NS} \\
\mathrm{R}=0.38^{* * *} \\
\mathrm{NS} \\
\mathrm{NS}\end{array}$ \\
\hline $\begin{array}{l}{[63]} \\
\text { Pinikahana \& } \\
\text { Happell } \\
(2004)\end{array}$ & CS & $N=136$ & MBI-HSS & $\begin{array}{l}\text { Nursing Stress Scale } \\
\text { Job Satisfaction Scale of nurse stress scale }\end{array}$ & $\begin{array}{l}\text { NR } \\
\text { NR }\end{array}$ & $\begin{array}{l}\text { NR } \\
\text { NR }\end{array}$ & $\begin{array}{l}\text { NR } \\
\text { NR }\end{array}$ \\
\hline $\begin{array}{l}{[64]} \\
\text { Priebe et al } \\
\text { (2005) }\end{array}$ & CS & $\mathrm{N}=109$ & MBI-HSS & $\begin{array}{l}\text { Identity scale } \\
\text { - Type of professional }\end{array}$ & $\frac{\text { Burnout }}{\mathrm{F}=20.72^{* * *}}$ & - & - \\
\hline $\begin{array}{l}{[65]} \\
\text { Prosser et al. } \\
\text { (1997) }\end{array}$ & CS & $\mathrm{N}=121$ & MBI- HSS & $\begin{array}{l}\text { Job Diagnostic Survey } \\
\text { - Stress from role (increased) } \\
\text { - Stress from work overload (increased) } \\
\text { - Satisfaction with career (decreased) } \\
\text { - Satisfaction from work with people (de- } \\
\text { creased) } \\
\text { - Children at home (reduced) } \\
\text { - Stress from poor support (increased) } \\
\text { - Stress from client (increased) } \\
\text { - Career satisfaction (decreased) } \\
\text { - Manager satisfaction (decreased) } \\
\text { - Satisfaction with career (increased) }\end{array}$ & $\begin{array}{l}\beta=4.1^{* * * *} \\
\beta=4.4^{* * *} \\
\beta=2.5^{* *} \\
\text { NS } \\
\text { NS } \\
\text { NS } \\
\text { NS } \\
\text { NS } \\
\text { NS } \\
\text { NR }\end{array}$ & $\begin{array}{l}\text { NS } \\
\text { NS } \\
\beta=1.1^{*} \\
\beta=1.1^{*} \\
\beta=3.0^{* * *} \\
\beta=1.1^{* *} \\
\beta=1.5^{* *} \\
\beta=1.4^{* *} \\
\beta=1.3^{*} \\
\text { NR }\end{array}$ & $\begin{array}{l}\text { NS } \\
\text { NS } \\
\text { NS } \\
\text { NS } \\
\text { NS } \\
\text { NS } \\
\text { NS } \\
\text { NS } \\
\text { NS } \\
\beta=2.6^{* * *}\end{array}$ \\
\hline $\begin{array}{l}{[66]} \\
\text { Prosser et al. } \\
\text { (1999) }\end{array}$ & LS & $\begin{array}{l}1994 \\
N=120 \\
1995 \\
N=166 \\
1996 \\
N=94\end{array}$ & MBI-HSS & $\begin{array}{l}\text { Higher if: } \\
\text { Nurse } \\
\text { Social worker } \\
\text { White } \\
\text { Lower if: } \\
\text { Very new } \\
\text { Very experienced in profession } \\
\text { In the sector longest community } \\
\text { Psychologist }\end{array}$ & $\begin{array}{l}\beta=4.03^{*} \\
\beta=13.32^{* * *} \\
\text { NS } \\
\beta=-3.24^{*} \\
\beta=-4.56^{* *} \\
\beta=-0.34^{*} \\
\text { NS }\end{array}$ & $\begin{array}{l}\beta=2.34^{* *} \\
\text { NS } \\
\beta=3.44^{* * *} \\
\text { NS } \\
\beta=-3.05^{*} \\
\text { NS } \\
\beta=-3.22^{* *}\end{array}$ & $\begin{array}{l}\text { NS } \\
\text { NS } \\
\text { NS } \\
\text { NS } \\
\text { NS } \\
\text { NS } \\
\text { NS }\end{array}$ \\
\hline $\begin{array}{l}\text { [67] Rogala } \\
\text { et al. (2016) } \\
\& \\
\text { (68)Shoji } \\
\text { et al. (2015) }\end{array}$ & LS & $\begin{array}{l}\text { Study } 1 \\
\text { T1: } 294 \\
\text { T2: } 135 \\
\text { Study } 2\end{array}$ & OLBI & $\begin{array}{l}\text { Multidimensional Scale of Perceived Social } \\
\text { Support } \\
\text { Disengagement T1 } \\
\text { Disengagement T2 } \\
\text { Self Efficacy T1 }\end{array}$ & $\begin{array}{l}\text { Emotional Exhaustion } \\
\text { T1 T2 } \\
\mathrm{r}=\mathrm{r}= \\
0.77^{* * *} 0.80^{* * *} \\
0.49^{* * *} 0.66^{* * *}\end{array}$ & $\begin{array}{l}\text { Depe } \\
\text { T1 T2 } \\
r=r= \\
N R \\
N R\end{array}$ & sonalisation \\
\hline
\end{tabular}


Table 2 (Continued)

\begin{tabular}{|c|c|c|c|c|c|c|c|}
\hline Study & Design & $\begin{array}{l}\text { No of } \\
\text { Resp }\end{array}$ & $\begin{array}{l}\text { Measure of } \\
\text { Burnout }\end{array}$ & Measure of determinants & $\mathrm{EE}$ & $\mathrm{DP}$ & PA \\
\hline & & $\begin{array}{l}\text { T1: } 306 \\
\text { T2: } 193\end{array}$ & & $\begin{array}{l}\text { Self Efficacy T2 } \\
\text { Social support T1 } \\
\text { Social support T2 } \\
\text { Exhaustion at T1 led to disengagement at T2 } \\
\text { Traumatic stress at T2 } \\
\text { Secondary Traumatic Stress (Secondary } \\
\text { Traumatic Stress Scale Scale (STSS)) } \\
\text { Job burnout at T1 led to STS } \\
\text { STS assessed at T1 did not lead to job burnout } \\
\text { at T2 }\end{array}$ & $\begin{array}{l}-0.52^{* * *} 0.38^{* * *} \\
-0.52^{* * *} 0.61^{* * *} \\
-0.29^{* * *} 0.23^{* * *} \\
-0.29^{* * *} 0.30^{* * *} \\
\text { p }<0.001 \\
\text { NS }\end{array}$ & $\begin{array}{l}\text { NR } \\
\text { NR } \\
\text { NR } \\
\text { NR } \\
\text { NR } \\
\text { NR }\end{array}$ & \\
\hline $\begin{array}{l}{[69]} \\
\text { Rossi et al. } \\
(2012)\end{array}$ & CS & $\mathrm{N}=260$ & $\begin{array}{l}\text { Professional } \\
\text { Quality of Life- III }\end{array}$ & $\begin{array}{l}\text { Marital status: separate, divorced, widowed } \\
\text { v's single } \\
\text { No of years spent in mental health } \\
\text { department } \\
\text { Lifetime traumatic events } \\
>1 \text { event v's none } \\
\text { Distress (GHQ-12 } \\
\text { score }>3 \text { ) }\end{array}$ & $\begin{array}{l}\text { Burnout } \\
r=3.117^{*} \\
r=0.099^{*} \\
r=3.154^{*} \\
r=-4.298^{* * *}\end{array}$ & $\begin{array}{l}- \\
- \\
-\end{array}$ & $\begin{array}{l}- \\
- \\
-\end{array}$ \\
\hline $\begin{array}{l}{[70]} \\
\text { Rupert and } \\
\text { Kent } \\
(2007)\end{array}$ & CS & $N=595$ & $\begin{array}{l}\text { MBI- HSS } \\
\text { Psychologist } \\
\text { Burnout } \\
\text { Inventory- } \\
\text { revised (PBI-R) }\end{array}$ & $\begin{array}{l}\text { Age } \\
\text { Total hours per week } \\
\text { PBI-R } \\
\text { - Negative clientele } \\
\text { - Over-involvement } \\
\text { - Control } \\
\text { - Support } \\
\text { Direct pay client } \\
\text { Managed care client } \\
\text { Job satisfaction scale } \\
\text { Career sustaining behaviours }\end{array}$ & $\begin{array}{l}r=-0.18^{*} \\
r=0.27^{*} \\
r=0.19^{*} \\
r=0.35^{*} \\
r=-0.29^{*} \\
\text { NS } \\
r=-0.11^{*} \\
\text { NS } \\
\text { NR } \\
\text { NS }\end{array}$ & $\begin{array}{l}r=-0.12^{*} \\
r=0.16^{*} \\
r=0.27^{*} \\
r=0.15^{*} \\
r=-0.22^{*} \\
\text { NS } \\
\text { NS } \\
\text { NS } \\
\text { NR } \\
r=-0.12^{*}\end{array}$ & $\begin{array}{l}r=0.12^{*} \\
N S \\
N S \\
r=0.16^{*} \\
r=0.42^{*} \\
r=0.11^{*} \\
r=0.13^{*} \\
\text { NS } \\
\text { NR } \\
r=0.30^{*}\end{array}$ \\
\hline $\begin{array}{l}{[71]} \\
\text { Rupert \& } \\
\text { Morgan } \\
(2005)\end{array}$ & $\mathrm{CS}$ & $\mathrm{N}=571$ & $\begin{array}{l}\text { MBI- HSS } \\
\text { Psychologists } \\
\text { Burnout } \\
\text { Inventory (PBI-R) }\end{array}$ & $\begin{array}{l}\text { Age } \\
\text { Total hours per week } \\
\text { PBI-R subscales } \\
\text { - negative clientele } \\
\text { - over-involvement } \\
\text { - control }\end{array}$ & $\begin{array}{l}-0.17^{*} \\
0.29^{*} \\
0.30^{*} \\
0.30^{*} \\
-0.29 \\
-0.23^{*} \\
0.15^{*}\end{array}$ & $\begin{array}{l}-0.13^{*} \\
\text { NS } \\
0.29^{*} \\
0.15^{*} \\
-0.21^{*} \\
\text { NS } \\
-0.14^{*}\end{array}$ & $\begin{array}{l}\text { NS } \\
\text { NS } \\
\text { NS } \\
0.18^{*} \\
0.33^{*} \\
\text { NS } \\
0.11^{*}\end{array}$ \\
\hline $\begin{array}{l}{[72]} \\
\text { Salyers et al. } \\
(2015)\end{array}$ & CS & $N=113$ & MBI - HSS & $\begin{array}{l}\text { Direct pay client } \\
\text { Managed care client } \\
\text { Sources of satisfaction and stress scale } \\
\text { Job diagnostic survey } \\
\text { Quality of care (quality of care scale } \\
\text { developed by } \\
\text { authors) } \\
\text { - Total score } \\
\text { - General work conscientiousness } \\
\text { - Client-centred care }\end{array}$ & $\begin{array}{l}r=-0.62^{* * *} \\
N S \\
r=-0.22^{*} \\
N S \\
r=0.58^{* * *} \\
r=0.51^{* * *}\end{array}$ & $\begin{array}{l}r=0.50^{* * *} \\
\text { NS } \\
r=-0.24^{*} \\
\text { NS } \\
\text { NS } \\
\text { NS }\end{array}$ & $\begin{array}{l}r=-0.44^{* * *} \\
r=0.5^{*} \\
\text { NS } \\
r=0.53^{* * *} \\
\text { NS } \\
\text { NS }\end{array}$ \\
\hline $\begin{array}{l}{[73]} \\
\text { Salyers et al. } \\
(2013) \\
{[74]} \\
\text { Sherring \& } \\
\text { Knight } \\
(2009)\end{array}$ & CS & $\begin{array}{l}N=152 \\
N=172\end{array}$ & $\begin{array}{l}\text { MBI- HSS } \\
\text { MBI-HSS }\end{array}$ & $\begin{array}{l}\text { Turnover intent past } 6 \text { months } \\
\text { Turnover intent next } 6 \text { months } \\
\text { Job Diagnostic Survey } \\
\text { Consumer Optimism Scale } \\
\text { Veteran association vs community providers } \\
\text { Clinical supervision monthly v's every } 2 / 3 \\
\text { months } \\
\text { Mental Health Nursing Questionnaire } \\
\text { - Feeling valued at work } \\
\text { - Perceived support at work } \\
\text { - Perceptions of involvement in the deci- } \\
\text { sion-making process regarding nursing } \\
\text { issues } \\
\text { - Perceptions of feeling involved in the } \\
\text { decision making process regarding } \\
\text { changes }\end{array}$ & $\begin{array}{l}\mathrm{NR} \\
\mathrm{NR} \\
\mathrm{t}=2.48^{*} \\
\mathrm{~F}=4.25^{* *} \\
\mathrm{~F}=16.82^{* * *} \\
\text { Effect size eta }{ }^{2}=0.29 \\
\mathrm{~F}=3.488^{* *} \\
\text { Effect size eta }{ }^{2}=0.08 \\
\mathrm{~F}=9.60^{* * *} \\
\text { Effect size eta }{ }^{2}=0.02 \\
\mathrm{~F}=8.06^{* * *} \\
\text { Effect size eta }{ }^{2}=0.17\end{array}$ & $\begin{array}{l}\text { NR } \\
\text { NR } \\
N S \\
N R \\
N R \\
N R \\
F=3.83^{* *} \\
\text { Effect size } \\
\text { eta }^{2}=0.09 \\
\text { NR }\end{array}$ & $\begin{array}{l}\mathrm{NR} \\
\mathrm{NR} \\
\mathrm{t}=6.29^{* * *} \\
\mathrm{NR} \\
\mathrm{F}=2.55^{*} \\
\text { Effect size } \\
\text { eta }^{2}=0.06 \\
\mathrm{NR} \\
\mathrm{NR} \\
\mathrm{NR}\end{array}$ \\
\hline $\begin{array}{l}{[75]} \\
\text { Siebert } \\
(2006)\end{array}$ & CS & $N=751$ & MBI- EE only & $\begin{array}{l}\text { Personal and Occupational variables- tool } \\
\text { developed for study } \\
\text { Marital status: Not married vs married } \\
\text { Living alone vs living with others } \\
\text { Employed by not private for profit } \\
\text { organisation vs private for profit organisation }\end{array}$ & $\begin{array}{l}\mathrm{F}=3.92^{*}(\mathrm{DF}=1) \\
\mathrm{F}=4.41^{*}(\mathrm{DF}=1) \\
\mathrm{F}=33.38^{* * *}(\mathrm{DF}=1) \\
\mathrm{F}=7.30^{*}(\mathrm{DF}=1)\end{array}$ & $\begin{array}{l}- \\
- \\
-\end{array}$ & $\begin{array}{l}- \\
- \\
-\end{array}$ \\
\hline $\begin{array}{l}{[77]} \\
\text { Sorgaard } \\
\text { et al. } \\
(2007)\end{array}$ & LS & $\mathrm{N}=414$ & MBI-HSS & $\begin{array}{l}\text { OSCAR Demographic questionnaire } \\
\text { Mental Health Professional Stress Scale } \\
\text { (MHPSS) }\end{array}$ & $\begin{array}{l}\text { NR } \\
\text { NR } \\
\text { NR } \\
\text { NR }\end{array}$ & $\begin{array}{l}\text { NR } \\
\text { NR } \\
\text { NR } \\
\text { NR }\end{array}$ & $\begin{array}{l}\text { NR } \\
\text { NR } \\
\text { NR } \\
\text { NR }\end{array}$ \\
\hline
\end{tabular}


Table 2 (Continued)

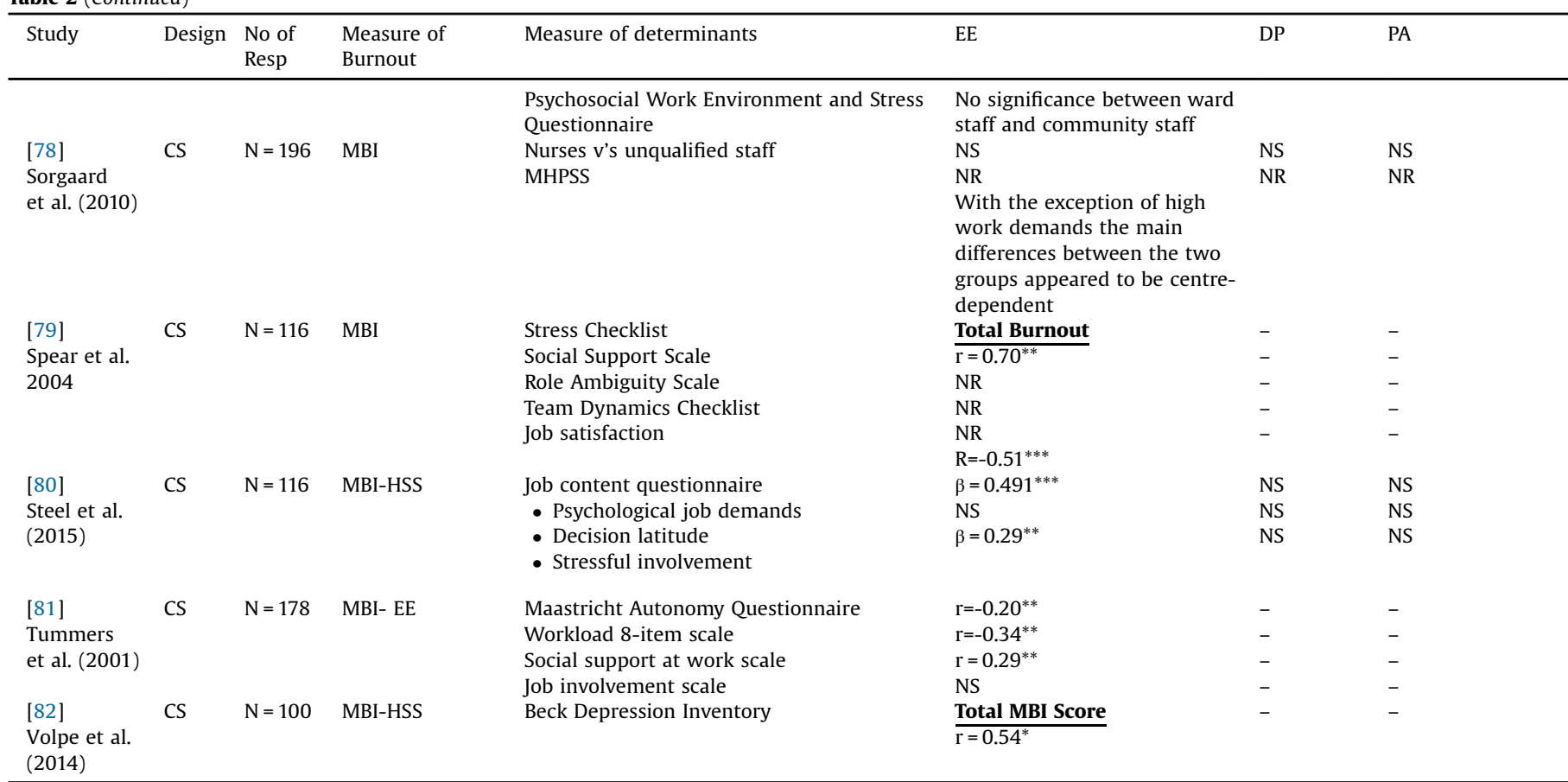

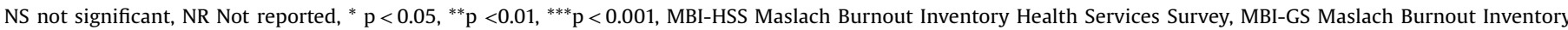

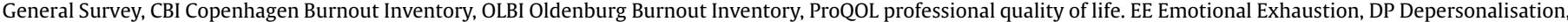

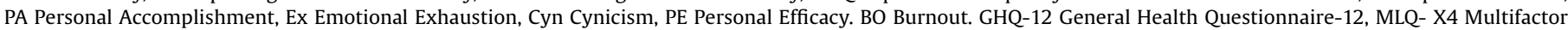

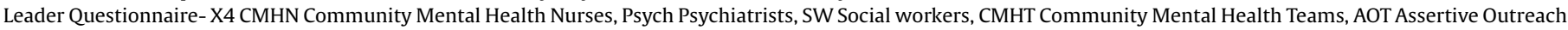
Team, CRT Crisis Resolution Team.

\subsection{Determinants of burnout in MHPs}

Fifty-nine studies were included in the narrative review of determinants. For this review, we categorised these determinants in terms of 'individual' factors and 'work-related' factors [3]. It was not possible to synthesise these results utilising meta-analytic techniques due to the variation in how determinants were assessed, and results reported. The studies and associated determinants are summarised in Table 2.

\subsubsection{Individual factors}

A negative correlation between age and depersonalisation was reported in eight studies [16,23-26,28,45,52,70,71]. While, two studies reported a positive relationship between age and depersonalisation [44,62]. A negative correlation between age and emotional exhaustion was reported by five studies $[26,28,45,70,71]$ and four studies reported a positive relationship between age and rating higher on the personal accomplishment sub-scale $[25,39,55,70]$. The findings on the relationship between gender and burnout dimensions were inconsistent. No consistent relationship between the length of service and burnout was found in the studies identified in this review

\subsubsection{Work-related factors}

3.7.2.1. Workload. Increased workload/ high caseloads were found consistently by the studies in this review to be associated with higher rates of burnout [16,21,33,36,39,50,51,53,56,65,81].

3.7.2.2. Job control. A sense of autonomy at work and perceived capacity to influence decisions that affect work was consistently reported by the studies identified in this review to be associated with lower rates of burnout, particularly lower rates of emotional exhaustion and increased rates of professional accomplishment [35,45,54,55,70,71,74,80,81].

3.7.2.3. Community. Community relates to the on-going relationships that employees have with other people on the job. Role conflict was found in this review to be associated with increased rates of emotional exhaustion [48,53,62], role ambiguity associated with increased emotional exhaustion [48,53] and role clarity was associated with higher rates of personal accomplishment [38]. Johnson et al. 2012 in their large sample of MHPs in the UK found that support from colleagues and managers was associated with reduced emotional strain and increased work engagement [45]. Lack of /inadequate clinical supervision was associated with increased risk of burnout in three studies [16,32,74]. In a sample of 189 community mental health nurses, Edwards et al. [31] demonstrated that higher scores on the Manchester Clinical Supervision Scale were associated with lower levels of measured burnout (EE: $r=-0.148, p<0.05$, DP $r=-0.22$, $\mathrm{p}=0.03$ ) [32]. Furthermore, Sherring \& Knight (2009) reported that in a population of 172 nurses those who reported a lesser quantity $(\mathrm{F}=4.25, \mathrm{p}=0.001)$ and or perceived inadequacy of clinical supervision $(\mathrm{F}=7.63, \mathrm{p}<0.001)$ reported higher rates of emotional exhaustion. Fairness in how staff feel they are treated and a sense of being rewarded for work was identified as being important in protecting against the development of burnout $[56,59]$.

3.7.2.4. Work setting. In a longitudinal study, comparing levels of burnout and sources of stress among the community and acute ward staff in six European countries Sorgaard et al. $2007(\mathrm{n}=414)$ found that burnout was not a serious problem among community or ward staff in this study at baseline, six months or 12 months [78]. However, they did find that rates of emotional exhaustion 
a:Mean score on Maslach Burnout inventory- Emotional Exhaustion subscale

Emotional Exhaustion

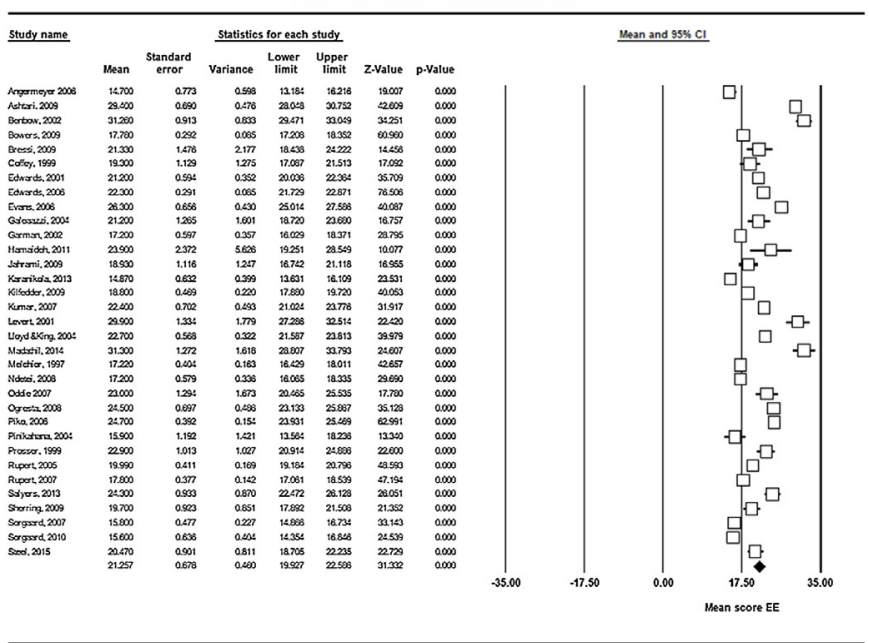

Meta Analysis c: Mean score on Maslach Burnout inventory- Personal Accomplishment subscale

Personal Accomplishment

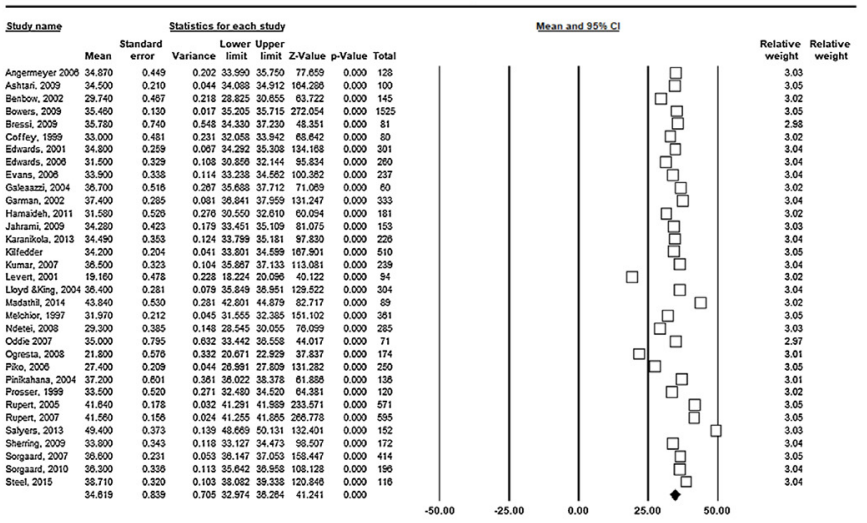

Meto Analysis

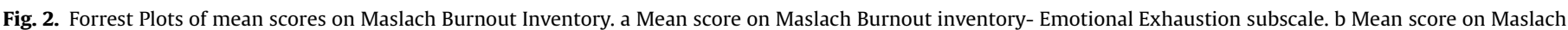
Burnout inventory- Depersonalisation subscale. c: Mean score on Maslach Burnout inventory- Personal Accomplishment subscale.

were higher in community staff (EE mean $18.31+/-10.5$ ) when compared to staff based on inpatient units (EE mean $15.8+/-9.74$ ) and that the variable that primarily distinguished between ward staff and community staff was job control. Furthermore, although the staff in the community reported a greater sense of control, they also reported higher work demands. Johnson et al. 2012 reported significant differences in work demand and job control described by staff working in different parts of the mental health service [45]. Staff working in community mental health teams reported the highest level of work demand (Mean 3.36 (SD 1.03), max score 5.0) while staff working staff working on rehabilitation wards reported the lowest level (Mean 2.47 (SD 0.94)). Conversely, staff in community mental health teams reported the highest level of job control (Mean 3.65 (SD 0.76), max score 5.0) while those working on acute general wards reported the lowest level (Mean 2.99 (SD $0.89)$ ). Furthermore, emotional exhaustion was significantly higher among acute general ward (EE mean 21.1, SD 12.7) and community mental health team staff (EE mean 23.8, SD 11.0) when compared to other service types $(F=8.87, p<0.0005)$. Nelson et al. 2009 $(n=433)$ assessed and compared the burnout levels of crisis resolution teams with assertive outreach and community mental health teams utilising a multicentre cross-sectional survey in England [57]. This study found that staff on crisis resolution and assertive outreach teams reported significantly higher sense of personal accomplishment than staff working in community mental health teams $(p=0.0005)$. Nelson et al. 2009 proposed that although the demands of working in a crisis resolution team are likely to be high, these may be mitigated by the sense of autonomy staff report and the benefit of working in a cohesive team [57]. Billings et al. $2003(n=301)$ compared satisfaction and burnout between assertive outreach teams and community mental health teams in London [24]. They found that staff on the assertive outreach team reported lower rates of depersonalisation $(\mathrm{r}=-1.7$, $p=0.01)$ and higher rates of personal accomplishment $(r=1.8$ $p=0.01$ ) compared to staff on the community mental health teams.

3.7.2.5. Professional background. Six studies reported on associations between burnout and MHPs professional background $[24,36,45,51,57,60,66]$. Five of these studies were completed in the UK. Johnson et al. 2012 reported that in their large 
a:Prevalence of Emotional Exhaustion

Prevalence of emotional exhaustion

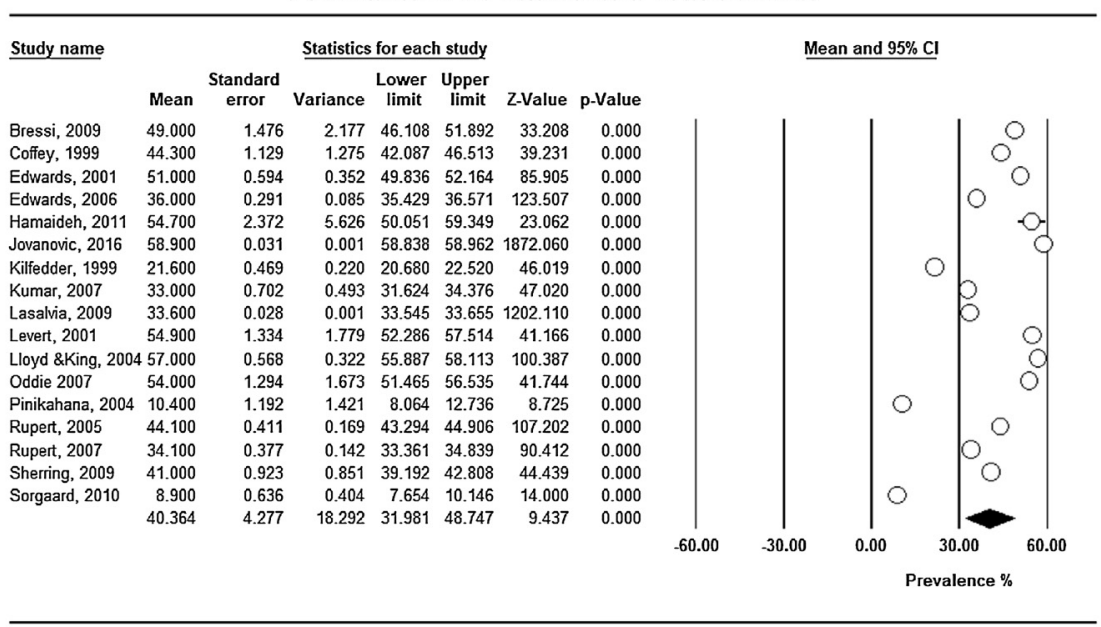

Meta Analysis

$\mathrm{b}$ : Prevalence of depersonalisation

Prevalence of depersonalisation

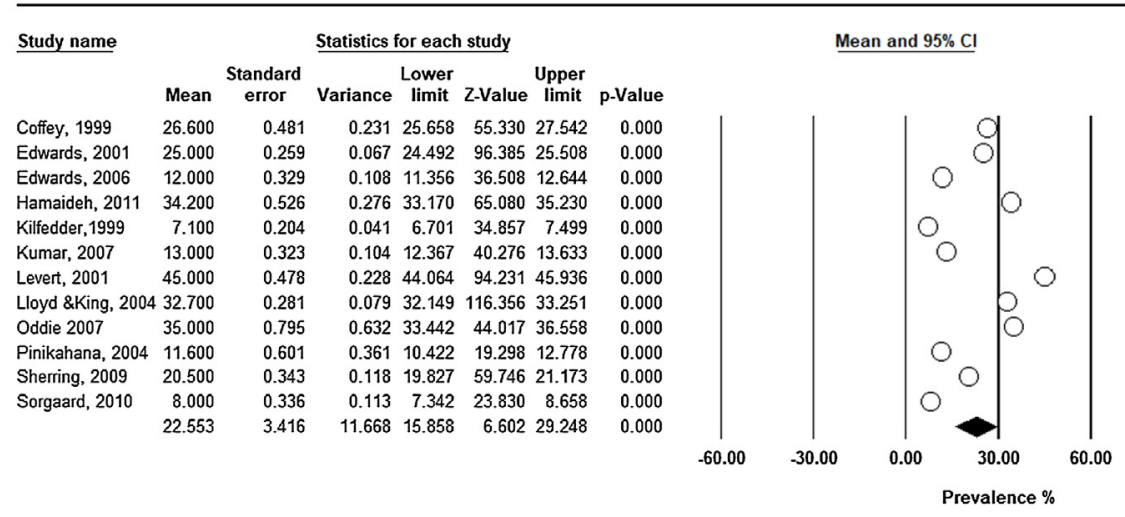

Meta Analysis

c:Prevalence of personal accomplishment

\section{Prevalence of personal accomplishment}

\begin{tabular}{|c|c|c|c|c|c|c|c|c|c|c|c|c|}
\hline \multirow[t]{2}{*}{ Study name } & \multicolumn{7}{|c|}{ Statistics for each study } & \multicolumn{5}{|c|}{ Mean and $95 \% \mathrm{Cl}$} \\
\hline & Mean & $\begin{array}{l}\text { Standard } \\
\text { error }\end{array}$ & Variance & $\begin{array}{c}\text { Lower } \\
\text { limit }\end{array}$ & $\begin{array}{c}\text { Upper } \\
\text { limit }\end{array}$ & Z-Value & p-Value & & & & & \\
\hline Edwards, 2001 & 14.000 & 0.375 & 0.140 & 13.266 & 14.734 & 37.368 & 0.000 & & & & & \\
\hline Edwards, 2006 & 10.000 & 0.335 & 0.112 & 9.344 & 10.656 & 29.860 & 0.000 & & & 0 & & \\
\hline Hamaideh, 2011 & 38.700 & 0.856 & 0.733 & 37.022 & 40.378 & 45.196 & 0.000 & & & & 0 & \\
\hline Kilfedder, 1999 & 33.100 & 0.350 & 0.122 & 32.414 & 33.786 & 94.621 & 0.000 & & & & & \\
\hline Kumar, 2007 & 23.800 & 0.344 & 0.118 & 23.126 & 24.474 & 69.161 & 0.000 & & & 3 & & \\
\hline Levert, 2001 & 3.300 & 0.852 & 0.726 & 1.630 & 4.970 & 3.873 & 0.000 & & & O & & \\
\hline Lloyd \&King, 2004 & 36.400 & 0.321 & 0.103 & 35.770 & 37.030 & 113.331 & 0.000 & & & & 0 & \\
\hline Oddie 2007 & 15.000 & 0.973 & 0.947 & 13.093 & 16.907 & 15.414 & 0.000 & & & 0 & & \\
\hline Pinikahana, 2004 & 11.600 & 1.012 & 1.024 & 9.617 & 13.583 & 11.464 & 0.000 & & & 0 & & \\
\hline Sherring, 2009 & 21.700 & 0.579 & 0.336 & 20.564 & 22.836 & 37.446 & 0.000 & & & 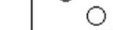 & & \\
\hline \multirow[t]{4}{*}{ Sorgaard, 2010} & 25.400 & 0.529 & 0.279 & 24.364 & 26.436 & 48.054 & 0.000 & & & & & \\
\hline & 21.192 & 3.358 & 11.276 & 14.610 & 27.773 & 6.311 & 0.000 & & & & & \\
\hline & & & & & & & & -60.00 & -30.00 & 0.00 & 0.00 & 60.00 \\
\hline & & & & & & & & & & & & \\
\hline
\end{tabular}

Meta Analysis

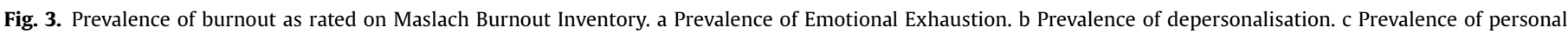
accomplishment. 
sample of MHPs ( $n=2258)$ social workers were significantly more likely than other MHP's to report high rates of emotional exhaustion $(\mathrm{F}=6.65, \mathrm{p}<0.001)$. In a longitudinal study, Prosser et al. 1999 reported higher rates of emotional exhaustion in social workers $(\beta=13.32, \mathrm{p}<0.01)$ and nurses $(\beta=4.03, \mathrm{p}<0.05)$ and lower rates of depersonalisation in psychologists $(\beta=-3.22$, $\mathrm{p}<0.01)$ [45,65]. Billings et al. 2003 and Nelson et al. 2009 also reported lower levels of depersonalisation in psychologists when compared to other MHPs $(\mathrm{r}=-3.2, \mathrm{p}<0.001 ; \mathrm{p}<0.05)[24,57]$.

\section{Discussion}

\subsection{Key findings}

This review included data on prevalence and determinants of burnout in MHPs from 62 studies, across 33 different countries. It is the first systematic review and meta-analysis on this topic in MHPs.

The overall estimate of the means for the burnout dimensions as rated on the MBI-HSS were 21.11 for emotional exhaustion, 6.76 for depersonalisation and 34.60 for depersonalisation. These means indicate that the average MHP has a 'high' level of emotional exhaustion, a 'moderate' level of depersonalisation but retains a 'high' level of personal accomplishment. These findings suggest that MHPs may still feel competent despite feeling exhausted, overextended, depleted and disconnected. The prevalence estimates for emotional exhaustion was $40 \%$ (range, $8 \%-59 \%$ ), for depersonalisation was $22 \%$ (range, $8 \%-65 \%$ ) and for low sense of personal accomplishment were 19\% (range 3\%-38\%). Given that emotional exhaustion is typically considered the core dimension of burnout, this review indicates that $40 \%$ of the respondents in the selected studies suffered from professional burnout [7].

The systematic review of determinants found a reasonably consistent relationship between increasing age and increased risk of depersonalisation but also an increased sense of personal accomplishment. The relationship between increased workload and increased rates of burnout was consistent across the studies identified. This relationship arose as a particular issue for those working in general community teams more than those working in specialist teams, e.g., assertive outreach teams, crisis teams, forensic settings. A sense of autonomy and perceived capacity to influence decisions at work were associated with lower rates of burnout. The data from the present study suggests that staff working in general adult in-patient settings report a lower sense of autonomy at work, while staff in the community teams and particularly in the specialist teams reported a greater sense of autonomy and associated personal accomplishment. The data identified in this review indicates that when relationships at work are characterised by role conflict, role ambiguity, and unresolved conflict, there is a higher risk of burnout. Clinical supervision, a sense of being treated fairly and of receiving fair reward for one's work appears to be protective. There was some data suggesting that social workers, working in the UK were at higher risk of burnout in comparison to other MHPs. Whereas, there was data suggesting that psychologists in the UK may be at lower risk of depersonalisation when compared to other MHPs.

\subsection{Comparison with previous literature}

The pooled estimates of respondents exceeding the 'high' cutoffs for the different dimensions of burnout are double those seen in the general population [92] and considerably higher than those reported in a systematic review of burnout in emergency nurses in which $26 \%$ reported high rates of emotional exhaustion [93,94], and a meta-analysis of health professionals working in palliative care in which $17.5 \%$ reported high rates of emotional exhaustion,
6.5\% reported high levels of depersonalisation and 19.5\% reported low levels of personal accomplishment [95]. The rates of emotional exhaustion and depersonalisation are also similar to those reported in a meta-analysis of burnout in cancer professionals, which reported high rates of emotional exhaustion in 36\%, high rates of depersonalisation in $34 \%$. However, the rates of low personal accomplishment in this meta-analysis of cancer professionals were reported as $25 \%$, which is considerably higher than the $18 \%$ reported in this meta-analysis of MHPs [96].

Consistent with previous reviews on this topic we did find significant relationships between workload, role conflict, lack of job control and burnout [7,15,88,97-99]. The findings that community staff are at higher risk of burnout is consistent with a literature review of burnout in community mental health nurses [100].

\subsection{Limitations}

This study has important limitations. Firstly, the levels of heterogeneity identified across studies in this review were high. However, meta-analyses of prevalence studies often report high levels of heterogeneity and published meta-analyses on the prevalence of burnout in other health professionals report similarly high levels of heterogeneity $[95,96,101]$. Some of the variance in this study was explained by the use of different cut-offs for 'caseness' on the MBI-HSS subscales, differences in the quality of the studies as rated on the Newcastle-Ottawa Scale, the average age of study participants, geographical region in which studies were conducted, sample sizes and \% of nurses/psychologists in the studies. However, work-related factors such as high caseload, poor team functioning, and lack of job control make MHPs more vulnerable to developing burnout. While these factors may be perpetuated by features common across the field of psychiatry; national health service characteristics and then local organisational factors are likely to be more critical to the work-related experience of MHPs and underlie their vulnerability to burnout. As such, some variation in the reported prevalence of the burnout phenomenon across countries and the world is unsurprising.

Secondly, although doctors, nurses, and psychologists were reasonably well represented in the studies identified, few studies reported individual data for other MHPs. Studies which reported on differences between rates of burnout in MHP's were primarily UK samples and given there are differences in how MHP's work in different countries these findings may not represent the experience in other countries and service delivery models. Thirdly, several conceptual models of burnout emphasise the need for a good person-environment fit to prevent burnout. However, the majority of studies identified only measured some work stressors and some outcomes, without taking into account the perception of the stressor by the MHP. These limitations mean that only a small part of the variance can be explained, interrelationships between determinants cannot be adequately investigated, results from different studies cannot be easily compared and causal relationships between determinants and outcomes cannot be made.

\section{Conclusion}

Burnout rates are high in MHPs, with the summary estimate of the prevalence of emotional exhaustion being $40 \%$. The present systematic review indicates that interventions to prevent and reduce burnout should focus on the promotion of professional autonomy, manageable caseloads, the development of good team function and the provision of quality clinical supervision to all MHPs.

Burnout rates are high in MHPs, with the summary estimate of the prevalence of emotional exhaustion being $40 \%$. The present 
systematic review indicates that interventions to prevent and reduce burnout should focus on the promotion of professional autonomy, manageable caseloads, the development of good team function and the provision of quality clinical supervision to all MHPs.

\section{Author contributions}

KOC, DMN and SP designed the study. KOC and DMN completed the data collection, analysis and interpretation. KOC drafted the article. DMN and SP revised the article. KOC, DMN and SP approved the final draft of the article.

\section{Conflict of interest statement}

\section{KOC, DMN and SP have no competing interest to declare.}

\section{Role of funding source}

This research did not receive funding from any specific grant from funding agencies in public, commercial, or not for profit sectors.

\section{Appendix A. Supplementary data}

Supplementary material related to this article can be found, in the online version, at doi:https://doi.org/10.1016/j. eurpsy.2018.06.003.

\section{References}

[1] Greene G.. A burnt-out case. New York: The Viking Press; 1961.

[2] Freudenberger HJ. Staff burn-out. J Soc Issues 1974;159-65.

[3] Maslach C, Schaufeli WB, Leiter MP. Job burnout. Annu Rev Psychol 2001;52:397-422.

[4] Maslach C, Jackson SE. Maslach burnout inventory. 1981.

[5] Kristensen TS, Borritz M, Villadsen E, Christensen KB. The Copenhagen burnout inventory: a new tool for the assessment of burnout. Work Stress 2005;192-207.

[6] Demerouti E. Burnout : eine folge konkreter arbeitsbedingungen bei dienstleistungs- und produktionstätigkeiten. 1999.

[7] Maslach C, Leiter MP. Understanding the burnout experience: recent research and its implications for psychiatry. World Psychiatry 2016;15(2):103-11.

[8] Demerouti E, Bakker AB, de Jonge J, Janssen PP, Schaufeli WB. Burnout and engagement at work as a function of demands and control. Scand J Work Environ Health 2001;27(4):279-86.

[9] Demerouti E, Mostert K, Bakker AB. Burnout and work engagement: a thorough investigation of the independency of both constructs. J Occup Health Psychol 2010;15(3):209-22.

[10] Schaufeli WB, Enzmann D. The burnout companion to study and practice: a critical analysis. London, UK: Taylor \& Francis; 1998.

[11] Ahola K, Väänänen A, Koskinen A, Kouvonen A, Shirom A. Burnout as a predictor of all-cause mortality among industrial employees: a 10-year prospective register-linkage study. J Psychosom Res 2010;69(1):51-7.

[12] Bakker AB, Le Blanc PM, Schaufeli WB. Burnout contagion among intensive care nurses. J Adv Nurs 2005;51(3):276-87.

[13] Westman M, Bakker AB, Roziner I, Sonnentag S. Crossover of job demands and emotional exhaustion within teams: a longitudinal multilevel study. Anxiety Stress Coping 2011;24(5):561-77.

[14] Demerouti E, Bakker AB, Leiter M. Burnout and job performance: the moderating role of selection, optimization, and compensation strategies. J Occup Health Psychol 2014;19(1):96-107.

[15] Rössler W. Stress, burnout, and job dissatisfaction in mental health workers. Eur Arch Psychiatry Clin Neurosci 2012;262(Suppl. 2):S65-9.

[16] Jovanović N, Podlesek A, Volpe U, Barrett E, Ferrari S, Rojnic Kuzman M, et al. Burnout syndrome among psychiatric trainees in 22 countries: risk increased by long working hours, lack of supervision, and psychiatry not being first career choice. Eur Psychiatry 2016;32:34-41.

[17] Cochran WG. The combination of estimates from different experiments. Biometrics 1954;10:101-29.

[18] Higgins JP, Thompson SG, Deeks JJ, Altman DG. Measuring inconsistency in meta-analyses. BMJ 2003;327(7414):557-60.

[19] Egger M, Smith GD. Misleading meta-analysis. BMJ 1995;311(7007):753-4.

[20] Egger M, Davey Smith G, Schneider M, Minder C. Bias in meta-analysis detected by a simple, graphical test. BMJ 1997;315(7109):629-34.
[21] Angermeyer MC, Bull N, Bernert S, Dietrich S, Kopf A. Burnout of caregivers: a comparison between partners of psychiatric patients and nurses. Arch Psychiatr Nurs 2006;20(4):158-65.

[22] Ashtari Z, Farhady Y, Khodaee MR. Relationship between job burnout and work performance in a sample of Iranian mental health staff. Afr J Psychiatry (Johannesbg) 2009;12(1):71-4.

[23] Benbow SM, Jolley DJ. Burnout and stress amongst old age psychiatrists. Int J Geriatr Psychiatry 2002;17(8):710-4.

[24] Billings J, Johnson S, Bebbington P, Greaves A, Priebe S, Muijen M, et al. assertive outreach teams in London: staff experiences and perceptions. PanLondon assertive outreach study, part 2. Br J Psychiatry 2003;183:139-47.

[25] Blau G, Tatum DS, Ward Goldberg C. Exploring correlates of burnout dimensions in a sample of psychiatric rehabilitation practitioners: a crosssectional study. Psychiatr Rehabil J 2013;36(3):166-72.

[26] Bowers L, Allan T, Simpson A, Jones J, Whittington R. Morale is high in acute inpatient psychiatry. Soc Psychiatry Psychiatr Epidemiol 2009;44(1):39-46.

[27] Bressi C, Porcellana M, Gambini O, Madia L, Muffatti R, Peirone A, et al. Burnout among psychiatrists in Milan: a multicenter survey. Psychiatr Serv 2009;60(7):985-8.

[28] Chakraborty R, Chatterjee A, Chaudhury S. Internal predictors of burnout in psychiatric nurses: an Indian study. Ind Psychiatry J 2012;21(2):119-24.

[29] Coffey M. Stress and burnout in forensic community mental health nurses: an investigation of its causes and effects. J Psychiatr Ment Health Nurs 1999;6 (6):433-43.

[30] Devilly GJ, Wright R, Varker T. Vicarious trauma, secondary traumatic stress or simply burnout? Effect of trauma therapy on mental health professionals. Aust N Z J Psychiatry 2009;43(4):373-85.

[31] Edwards D, Burnard P, Coyle D, Fothergill A, Hannigan B. A stepwise multivariate analysis of factors that contribute to stress for mental health nurses working in the community. J Adv Nurs 2001;36(6):805-13.

[32] Edwards D, Burnard P, Hannigan B, Cooper L, Adams J, Juggessur T, et al. Clinical supervision and burnout: the influence of clinical supervision for community mental health nurses. J Clin Nurs 2006;15(8):1007-15.

[33] Evans S, Huxley P, Gately C, Webber M, Mears A, Pajak S, et al. Mental health, burnout and job satisfaction among mental health social workers in England and Wales. Br J Psychiatry 2006;188:75-80.

[34] Fong TC, Ho RT, Au-Yeung FS, Sing CY, Law KY, Lee LF, et al. The relationships of change in work climate with changes in burnout and depression: a 2-year longitudinal study of Chinese mental health care workers. Psychol Health Med 2015;1-12.

[35] Garman AN, Corrigan PW, Morris S. Staff burnout and patient satisfaction: evidence of relationships at the care unit level. J Occup Health Psychol 2002;7 (3):235-41.

[36] Galeazzi GM, Delmonte S, Fakhoury W, Priebe S. Morale of mental health professionals in Community mental health services of a Northern Italian Province. Epidemiol Psichiatr Soc 2004:13(3):191-7.

[37] Green AE, Miller EA, Aarons GA. Transformational leadership moderates the relationship between emotional exhaustion and turnover intention among community mental health providers. Commun Ment Health J 2013;49 (4):373-9.

[38] Green AE, Albanese BJ, Shapiro NM, Aarons GA. The roles of individual and organizational factors in burnout among community-based mental health service providers. Psychol Serv 2014;11(1):41-9.

[39] Hamaideh SH. Burnout, social support, and job satisfaction among Jordanian mental health nurses. Issues Ment Health Nurs 2011;32(4):234-42.

[40] Happell B, Martin T, Pinikahana J. Burnout and job satisfaction: a comparative study of psychiatric nurses from forensic and a mainstream mental health service. Int J Ment Health Nurs 2003;12(1):39-47.

[41] Imai H, Nakao H, Nakagi Y, Niwata S, Sugioka Y, Itoh T, et al. Prevalence of burnout among public health nurses in charge of mental health services and emergency care systems in Japan. Environ Health Prev Med 2006;11(6):286 91.

[42] Imai H, Nakao H, Tsuchiya M, Kuroda Y, Katoh T. Burnout and work environments of public health nurses involved in mental health care. Occup Environ Med 2004;61(9):764-8.

[43] Jahrami H. A survey of burnout of the mental health occupational therapy staff in the psychiatric hospital, Bahrain. Br J Occup Ther 2009;458-64.

[44] Jeanneau M, Armelius K. Self-image and burnout in psychiatric staff. J Psychiatr Ment Health Nurs 2000;7(5):399-406.

[45] Johnson S, Osborn DP, Araya R, Wearn E, Paul M, Stafford M, et al. Morale in the English mental health workforce: questionnaire survey. Br J Psychiatry 2012;201(3):239-46.

[46] Johnson H, Worthington R, Gredecki N, Rachel Wilks-Riley F. The relationship between trust in work colleagues, impact of boundary violations and burnout amount staff within a forensic psychiatric service. J Forensic Pract 2016;6475.

[47] Karanikola MN, Papathanassoglou EE. Exploration of the burnout syndrome occurrence among mental health nurses in Cyprus. Arch Psychiatr Nurs 2013;27(6):319-26.

[48] Kilfedder CJ, Power KG, Wells TJ. Burnout in psychiatric nursing. J Adv Nurs 2001:34(3):383-96.

[49] Kumar S, Fischer J, Robinson E, Hatcher S, Bhagat RN. Burnout and job satisfaction in New Zealand psychiatrists: a national study. Int J Soc Psychiatry 2007;53(4):306-16. 
[50] Kumar S, Hatcher S, Dutu G, Fischer J, Ma’u E. Stresses experienced by psychiatrists and their role in burnout: a national follow-up study. Int J Soc Psychiatry 2011;57(2):166-79.

[51] Lasalvia A, Bonetto C, Bertani M, Bissoli S, Cristofalo D, Marrella G, et al. Influence of perceived organisational factors on job burnout: survey of community mental health staff. Br J Psychiatry 2009;195(6):537-44.

[52] Lloyd C, King R. A survey of burnout among Australian mental health occupational therapists and social workers. Soc Psychiatry Psychiatr Epidemiol 2004;39(9):752-7.

[53] Levert T, Lucas M, Ortlepp K. Burnout in psychiatric nurses: contributions of the work environment and a sense of coherence. South African J Psychol 2000;36-41.

[54] Madathil R, Heck NC, Schuldberg D. Burnout in psychiatric nursing: examining the interplay of autonomy, leadership style, and depressive symptoms. Arch Psychiatr Nurs 2014;28(3):160-6.

[55] Melchior ME, van den Berg AA, Halfens R, Huyer Abu-Saad H, Philipsen H, Gassman P. Burnout and the work environment of nurses in psychiatric longstay care settings. Soc Psychiatry Psychiatr Epidemiol 1997;32(3):158-64.

[56] Ndetei DM, Pizzo M, Maru H, Ongecha FA, Khasakhala LI, Mutiso V, et al. Burnout in staff working at the mathari psychiatric hospital. Afr J Psychiatry (Johannesbg) 2008;11(3):199-203.

[57] Nelson T, Johnson S, Bebbington P. Satisfaction and burnout among staff of crisis resolution, assertive outreach and community mental health teams. A multicentre cross sectional survey. Soc Psychiatry Psychiatr Epidemiol 2009;44(7):541-9.

[58] Oddie S, Ousley L. Assessing burn-out and occupational stressors in a medium secure service. . p. 32-48.

[59] Ogresta J, Rusac S, Zorec L. Relation between burnout syndrome and job satisfaction among mental health workers. Croat Med J 2008;49(3):364-74.

[60] Onyett S, Pillinger T, Muijen M. Job satisfaction and burnout amount members of community mental health teams. J Mental Health 1997;55-66.

[61] Oyefeso A, Clancy C, Farmer R. Prevalence and associated factors in burnout and psychological morbidity among substance misuse professionals. BMC Health Serv Res 2008;8:39.

[62] Piko BF. Burnout, role conflict, job satisfaction and psychosocial health among Hungarian health care staff: a questionnaire survey. Int J Nurs Stud 2006;43(3):311-8.

[63] Pinikahana J, Happell B. Stress, burnout and job satisfaction in rural psychiatric nurses: a Victorian study. Aust J Rural Health 2004:12(3):120-5.

[64] Priebe S, Fakhoury WK, Hoffmann K, Powell RA. Morale and job perception of community mental health professionals in Berlin and London. Soc Psychiatry Psychiatr Epidemiol 2005;40(3):223-32.

[65] Prosser D, Johnson S, Kuipers E, Szmukler G, Bebbington P, Thornicroft G. Perceived sources of work stress and satisfaction among hospital and community mental health staff, and their relation to mental health, burnout and job satisfaction. J Psychosom Res 1997:43(1):51-9.

[66] Prosser D, Johnson S, Kuipers E, Dunn G, Szmukler G, Reid Y, et al. Mental health, "burnout" and job satisfaction in a longitudinal study of mental health staff. Soc Psychiatry Psychiatr Epidemiol 1999;34(6):295-300.

[67] Rogala A, Shoji K, Luszczynska A, Kuna A, Yeager C, Benight CC, et al. From exhaustion to disengagement via self-efficacy change: findings from Two longitudinal studies among human services workers. Front Psychol 2015;6:2032.

[68] Shoji K, Lesnierowska M, Smoktunowicz E, Bock J, Luszczynska A, Benight CC, et al. What comes first, job burnout or secondary traumatic stress? Findings from Two longitudinal studies from the U.S. And Poland. PLoS One 2015;10(8) e0136730.

[69] Rossi A, Cetrano G, Pertile R, Rabbi L, Donisi V, Grigoletti L, et al. Burnout, compassion fatigue, and compassion satisfaction among staff in communitybased mental health services. Psychiatry Res 2012;200(2-3):933-8.

[70] Rupert PA, Kent JS. Gender and work setting differences in career-sustaining behaviors and burnout among professional psychologists. Prof Psychol Res Pract 2007;88-96.

[71] Rupert PA, Morgan DJ. Work setting and burnout amount professional psychologists. Prof Psychol: Res Pract 2005;544-50.

[72] Salyers MP, Flanagan ME, Firmin R, Rollins AL. Clinicians' perceptions of how burnout affects their work. Psychiatr Serv 2015;66(2):204-7.

[73] Salyers MP, Rollins AL, Kelly YF, Lysaker PH, Williams JR. Job satisfaction and burnout among VA and community mental health workers. Adm Policy Ment Health 2013;40(2):69-75.

[74] Sherring S, Knight D. An exploration of burnout among city mental health nurses. Br J Nurs 2009;18(20):1234-40.

[75] Siebert DC. Personal and occupational factors in burnout among practicing social workers. J Soc Serv Res 2006;25-44.
[76] Singh C, Cross W, Jackson D. Staff burnout-a comparative study of metropolitan and rural mental health nurses within Australia. Issues Ment Health Nurs 2015;36(7):528-37.

[77] Sørgaard KW, Ryan P, Hill R, Dawson I, Group O. Sources of stress and burnout in acute psychiatric care: inpatient vs. Community staff. Soc Psychiatry Psychiatr Epidemiol 2007;42(10):794-802.

[78] Sorgaard KW, Ryan P, Dawson I. Qualified and unqualified (N-R C) mental health nursing staff-minor differences in sources of stress and burnout. A European multi-centre study. BMC Health Serv Res 2010;10:163.

[79] Spear J, Wood L, Chawla S, Devis A, Nelson J. Job satisfaction and burnout in mental health services for older people. Australas Psychiatry 2004;12(1):5861.

[80] Steel C, Macdonald J, Schröder T, Mellor-Clark J. Exhausted but not cynical: burnout in therapists working within improving access to psychological therapy services. J Ment Health 2015;24(1):33-7.

[81] Tummers GE, Janssen PP, Landeweerd A, Houkes I. A comparative study of work characteristics and reactions between general and mental health nurses: a multi-sample analysis. J Adv Nurs 2001;36(1):151-62.

[82] Volpe U, Luciano M, Palumbo C, Sampogna G, Del Vecchio V, Fiorillo A. Risk of burnout among early career mental health professionals. J Psychiatr Ment Health Nurs 2014;21(9):774-81.

[83] Maslach C, Jackson SE, Leiter MP. The maslach burnout inventory manual. 3rd ed. Paolto Alto: Consulting Psychologists Press; 1996.

[84] Glisson C, Landsverk J, Schoenwald S, Kelleher K, Hoagwood KE, Mayberg S, et al. Assessing the organizational social context (OSC) of mental health services: implications for research and practice. Adm Policy Ment Health 2008;35(1-2):98-113

[85] Stamm BH. The concise ProQOL manual. 2nd ed Pocatello; 2010.

[86] Poghosyan L, Aiken LH, Sloane DM. Factor structure of the maslach burnout inventory: an analysis of data from large scale cross-sectional surveys of nurses from eight countries. Int J Nurs Stud 2009;46(7):894-902.

[87] Kitaoka-Higashiguchi K, Ogino K, Masuda S. [Validation of a Japanese research version of the maslach burnout inventory-General survey] Shinrigaku Kenkyu 2004;75(5):415-9.

[88] Paris M, Hoge MA. Burnout in the mental health workforce: a review. J Behav Health Serv Res 2010;37(4):519-28.

[89] Gil-Monte PR. Factorial validity of the maslach burnout inventory (MBI-HSS) among Spanish professionals. Rev Saude Publica. 2005;39(1):1-8.

[90] Schutte N, Toppinen S, Kalimo R, Schaufeli W. The factorial validity of the maslach burnout inventory- General survey (MBI-GS) across occupational groups and nations. J Occup Organ Psychol 2000;53-66.

[91] Hannigan B, Edwards D, Coyle D, Fothergill A, Burnard P. Burnout in community mental health nurses: findings from the all-Wales stress study. J Psychiatr Ment Health Nurs 2000;7(2):127-34.

[92] Lindblom KM, Linton SJ, Fedeli C, Bryngelsson IL. Burnout in the working population: relations to psychosocial work factors. Int J Behav Med 2006;13 (1):51-9.

[93] Adriaenssens J, De Gucht V, Maes S. Determinants and prevalence of burnout in emergency nurses: a systematic review of 25 years of research. Int J Nurs Stud 2015;52(2):649-61.

[94] Gómez-Urquiza JL, Aneas-López AB, Fuente-Solana EI, Albendín-García L, DíazRodríguez L, Fuente GA. Prevalence, risk factors, and levels of burnout among oncology nurses: a systematic review. Oncol Nurs Forum 2016;43(3):E104-20.

[95] Parola V, Coelho A, Cardoso D, Sandgren A, Apóstolo J. Prevalence of burnout in health professionals working in palliative care: a systematic review. JBI Database System Rev Implement Rep 2017;15(7):1905-33.

[96] Trufelli DC, Bensi CG, Garcia JB, Narahara JL, Abrão MN, Diniz RW, et al. Burnout in cancer professionals: a systematic review and meta-analysis. Eur J Cancer Care (Engl) 2008;17(6):524-31.

[97] Fothergill A, Edwards D, Burnard P. Stress, burnout, coping and stress management in psychiatrists: findings from a systematic review. Int J Soc Psychiatry 2004;50(1):54-65

[98] Morse G, Salyers MP, Rollins AL, Monroe-DeVita M, Pfahler C. Burnout in mental health services: a review of the problem and its remediation. Adm Policy Ment Health 2012;39(5):341-52.

[99] Kumar S. Burnout in psychiatrists. World Psychiatry 2007;6(3):186-9.

[100] Edwards D, Burnard P, Coyle D, Fothergill A, Hannigan B. Stress and burnout in community mental health nursing: a review of the literature. J Psychiatr Ment Health Nurs 2000;7(1):7-14.

[101] Gómez-Urquiza JL, De la Fuente-Solana EI, Albendín-García L, Vargas-Pecino C, Ortega-Campos EM, Cañadas-De la Fuente GA. Prevalence of burnout syndrome in emergency nurses: a meta-analysis. Crit Care Nurse 2017;37(5): e1-9. 